\title{
Proton and neutron electromagnetic form factors from lattice QCD
}

\author{
C. Alexandrou, ${ }^{1,2}$ S. Bacchio, ${ }^{1}$ M. Constantinou, ${ }^{3}$ J. Finkenrath, ${ }^{2}$ K. Hadjiyiannakou, ${ }^{2}$ K. Jansen, ${ }^{4}$ \\ G. Koutsou, ${ }^{2}$ and A. Vaquero Aviles-Casco ${ }^{5}$ \\ ${ }^{1}$ Department of Physics, University of Cyprus, P.O. Box 20537, 1678 Nicosia, Cyprus \\ ${ }^{2}$ Computation-based Science and Technology Research Center, The Cyprus Institute, \\ 20 Kavafi Street, Nicosia 2121, Cyprus \\ ${ }^{3}$ Department of Physics, Temple University, 1925 North 12th Street, Philadelphia, \\ Pennsylvania 19122-1801, USA \\ ${ }^{4}$ NIC, DESY, Platanenallee 6, D-15738 Zeuthen, Germany \\ ${ }^{5}$ Department of Physics and Astronomy, University of Utah, Salt Lake City, Utah 84112, USA
}

(Received 4 January 2019; revised manuscript received 4 May 2019; published 25 July 2019)

\begin{abstract}
The electromagnetic form factors of the proton and the neutron are computed within lattice QCD using simulations with quark masses fixed to their physical values. Both connected and disconnected contributions are computed. We analyze two new ensembles of $N_{f}=2$ and $N_{f}=2+1+1$ twisted mass clover-improved fermions and determine the proton and neutron form factors, the electric and magnetic radii, and the magnetic moments. We use several values of the sink-source time separation in the range of 1.0 to $1.6 \mathrm{fm}$ to ensure ground state identification. Disconnected contributions are calculated to an unprecedented accuracy at the physical point. Although they constitute a small correction, they are nonnegligible and contribute up to $15 \%$ for the case of the neutron electric charge radius.
\end{abstract}

DOI: 10.1103/PhysRevD.100.014509

\section{INTRODUCTION}

Nucleons being composite particles have a nontrivial internal structure that can be probed by measuring their electromagnetic form factors. These fundamental quantities have been extensively studied both theoretically and experimentally. However, open issues still persist and there are ongoing experimental efforts to determine them at higher precision and over a wider range of momentum transfers and to describe them theoretically. The proton electric form factor is extracted to high precision from electron proton scattering [1]. Its slope at vanishing momentum transfer squared yields the proton charge root-mean-square (rms) radius. Prior to 2010, the charge rms radius of the proton was considered a well-determined quantity (see Ref. [2] for a recent review). A pioneering experiment using Lamb shifts in muonic hydrogen surprisingly found a value smaller by 5 standard deviations [3], triggering the so-called proton radius puzzle. The origin of this discrepancy is not yet understood, and potential systematic uncertainties related to the analysis methodologies in the two types of experiments have not been

Published by the American Physical Society under the terms of the Creative Commons Attribution 4.0 International license. Further distribution of this work must maintain attribution to the author(s) and the published article's title, journal citation, and DOI. Funded by SCOAP ${ }^{3}$. excluded. Another quantity of interest is the neutron electric form factor [4], which is accessed indirectly experimentally through electron-deuteron or electron-helium scattering and therefore remains poorly known. It is of substantial importance to compute these fundamental quantities from first principles using lattice $\mathrm{QCD}$, which provides an ideal formulation for such an investigation and with simulations at physical values of the QCD parameters.

Within this work, we compute the proton and neutron electromagnetic form factors including light quark disconnected contributions. We use an ensemble of twisted mass fermions with two degenerate light quarks, a strange, and a charm quark $\left(N_{f}=2+1+1\right)$ with masses fixed to their physical value (referred to hereafter as physical point). A clover term is added to the action to suppress isospin breaking effects that come quadratically with the lattice spacing. Details on the simulation can be found in Ref. [5]. We will refer to this ensemble as cB211.072.64. In addition, we present an analysis of a twisted mass ensemble of two degenerate light quarks with masses fixed to their physical values $\left(N_{f}=2\right)$ to assess finite volume artifacts by comparing to previous results obtained using an $N_{f}=2$ ensemble with a smaller volume and same pion mass and lattice spacing [6,7]. Comparison between $N_{f}=2$ and $N_{f}=2+1+1$ also sheds light on any possible unquenching effect of the strange and charm quarks. The momentum dependence of the form factors is fitted using two Ansätze, namely either a dipole or the 
Galster-like parametrization [8] and the model-independent $z$-expansion [9]. The fits allow for the extraction of the magnetic moment and the electric and magnetic rms radii of the proton and neutron and provide a measure of the systematics due to the choice of the fit method.

A crucial component of our analysis is the use of hierarchical probing [10] combined with deflation of the lower-lying eigenvalues [11] that enables us to calculate the light quark disconnected contributions to the form factors at an unprecedented accuracy at the physical point. This allows us to obtain the proton and neutron form factors at the physical point without neglecting disconnected contributions.

The remainder of this paper is organized as follows: In Sec. II, we describe the nucleon matrix elements required to extract the electromagnetic form factors and in Sec. III we provide details on the lattice QCD techniques employed for the computation of the connected and disconnected diagrams. In Sec. IV, we discuss the analysis of the data paying particular attention to the identification of the ground state matrix element. In Sec. V we include an assessment of finite volume and unquenching effects using results from the analysis of the two $N_{f}=2$ ensembles. In Sec. VI, we fit the isovector and isoscalar form factors to extract the magnetic moments and radii. We compare to other lattice QCD studies using simulations close to physical pion masses in Sec. VII [12-15]. Our final results for the proton and neutron electromagnetic form factors are given in Sec. VIII. Finally, in Sec. IX, we summarize our findings and conclude. For completeness, we summarize in Appendix A the decomposition of the nucleon matrix elements in terms of the form factors and in Appendix B we provide a Table VI with the numerical results for the electric and magnetic form factors as a function of the momentum transfer squared.

\section{ELECTROMAGNETIC FORM FACTORS}

The nucleon matrix element of the electromagnetic current is parametrized in terms of the Dirac $\left(F_{1}\right)$ and Pauli $\left(F_{2}\right)$ form factors given in Minkowski space by

$$
\begin{aligned}
& \left\langle N\left(p^{\prime}, s^{\prime}\right)\left|j_{\mu}\right| N(p, s)\right\rangle \\
& =\sqrt{\frac{m_{N}^{2}}{E_{N}\left(\vec{p}^{\prime}\right) E_{N}(\vec{p})}} \\
& \quad \times \bar{u}_{N}\left(p^{\prime}, s^{\prime}\right)\left[\gamma_{\mu} F_{1}\left(q^{2}\right)+\frac{i \sigma_{\mu \nu} q^{\nu}}{2 m_{N}} F_{2}\left(q^{2}\right)\right] u_{N}(p, s) .
\end{aligned}
$$

$N(p, s)$ is the nucleon state with initial (final) momentum $p\left(p^{\prime}\right)$ and spin $s\left(s^{\prime}\right)$, with energy $E_{N}(\vec{p})\left(E_{N}\left(\vec{p}^{\prime}\right)\right)$ and mass $m_{N} \cdot q^{2} \equiv q_{\mu} q^{\mu}$ is the momentum transfer squared $q_{\mu}=\left(p_{\mu}^{\prime}-p_{\mu}\right)$ and $u_{N}$ is the nucleon spinor. The local vector current $j_{\mu}$ is given by

$$
j_{\mu}=\sum_{f} e_{f} j_{\mu}^{f}=\sum_{f} e_{f} \bar{q}_{f} \gamma_{\mu} q_{f}
$$

where $q_{f}$ is the quark field of flavor $f$ and $e_{f}$ its electric charge, and the summation runs over all the quark flavors. Instead of the local vector current, we instead use the symmetrized lattice conserved vector current given by

$$
\begin{aligned}
j_{\mu}^{f}(x)= & \frac{1}{4}\left[\bar{q}_{f}(x+\hat{\mu}) U_{\mu}^{\dagger}(x)\left(1+\gamma_{\mu}\right) q_{f}(x)\right. \\
& -\bar{q}_{f}(x) U_{\mu}(x)\left(1-\gamma_{\mu}\right) q_{f}(x+\hat{\mu}) \\
& +\bar{q}_{f}(x) U_{\mu}^{\dagger}(x-\hat{\mu})\left(1+\gamma_{\mu}\right) q_{f}(x-\hat{\mu}) \\
& \left.-\bar{q}_{f}(x-\hat{\mu}) U_{\mu}(x-\hat{\mu})\left(1-\gamma_{\mu}\right) q_{f}(x)\right],
\end{aligned}
$$

which, unlike the local vector current, does not need renormalization. The electric and magnetic Sachs form factors $G_{E}\left(q^{2}\right)$ and $G_{M}\left(q^{2}\right)$ are alternative Lorentz invariant quantities and are expressed in terms of $F_{1}\left(q^{2}\right)$ and $F_{2}\left(q^{2}\right)$ via the relations,

$$
\begin{gathered}
G_{E}\left(q^{2}\right)=F_{1}\left(q^{2}\right)+\frac{q^{2}}{4 m_{N}^{2}} F_{2}\left(q^{2}\right), \\
G_{M}\left(q^{2}\right)=F_{1}\left(q^{2}\right)+F_{2}\left(q^{2}\right) .
\end{gathered}
$$

In the isospin limit, where the up and down quarks are degenerate, we consider the isovector combination $\langle p| j_{\mu}^{u}-$ $j_{\mu}^{d}|p\rangle$ that gives the difference between the proton and neutron form factors and the isoscalar combination $\langle p| j_{\mu}^{u}+$ $j_{\mu}^{d}|p\rangle / 3$ for the sum of the proton and neutron form factors. The electric form factor at zero momentum yields the nucleon charge, i.e., $G_{E}^{p}(0)=1$ and $G_{E}^{n}(0)=0$ which, when using the lattice conserved current, holds by symmetry, even prior to gauge averaging. The magnetic form factor at $q^{2}=0$ gives the magnetic moment, while the radii can be extracted from the slope of the electric and magnetic form factors as $q^{2} \rightarrow 0$, namely,

$$
\left\langle r^{2}\right\rangle=\left.\frac{6}{G(0)} \frac{\partial G\left(q^{2}\right)}{\partial q^{2}}\right|_{q^{2}=0} .
$$

\section{CALCULATION ON THE LATTICE}

\section{A. Nucleon matrix element}

Extraction of nucleon matrix elements within the lattice QCD formulation requires the evaluation of two- and threepoint correlation functions in Euclidean space. We thus give all quantities in Euclidean space from here on. We use the standard nucleon interpolating field

$$
J_{N}(\vec{x}, t)=\epsilon^{a b c} u^{a}(x)\left[u^{b \top}(x) \mathcal{C} \gamma_{5} d^{c}(x)\right],
$$


where $u$ and $d$ are up- and down-quark spinors and $\mathcal{C}=$ $\gamma_{0} \gamma_{2}$ is the charge conjugation matrix. The two-point function in momentum space is given by

$$
\begin{aligned}
& C\left(\Gamma_{0}, \vec{p} ; t_{s}, t_{0}\right) \\
& =\sum_{\vec{x}_{s}} \operatorname{Tr}\left[\Gamma_{0}\left\langle J_{N}\left(t_{s}, \vec{x}_{s}\right) \bar{J}_{N}\left(t_{0}, \vec{x}_{0}\right)\right\rangle\right] e^{-i\left(\vec{x}_{s}-\vec{x}_{0}\right) \cdot \vec{p}},
\end{aligned}
$$

and the three-point function is given by

$$
\begin{aligned}
& C_{\mu}\left(\Gamma_{\nu}, \vec{q}, \vec{p}^{\prime} ; t_{s}, t_{\mathrm{ins}}, t_{0}\right) \\
& =\sum_{\vec{x}_{\mathrm{in} s}, \vec{x}_{s}} e^{i\left(\vec{x}_{\mathrm{ins}}-\vec{x}_{0}\right) \cdot \vec{q}} e^{-i\left(\vec{x}_{s}-\vec{x}_{0}\right) \cdot \vec{p}^{\prime}} \\
& \quad \times \operatorname{Tr}\left[\Gamma_{\nu}\left\langle J_{N}\left(t_{s}, \vec{x}_{s}\right) j_{\mu}\left(t_{\mathrm{ins}}, \vec{x}_{\mathrm{ins}}\right) \bar{J}_{N}\left(t_{0}, \vec{x}_{0}\right)\right\rangle\right] .
\end{aligned}
$$

The initial position and time, $x_{0}$, is referred to as the source, the position and time of the current $j_{\mu}$ couples to a quark is denoted by $x_{\text {ins }}$ and referred to as the insertion, and the final position, $x_{s}$, as the sink. $\Gamma_{\nu}$ is a projector acting on spin indices, with $\Gamma_{0}=\frac{1}{2}\left(1+\gamma_{0}\right)$ yielding the unpolarized and $\Gamma_{k}=\Gamma_{0} i \gamma_{5} \gamma_{k}$ the polarized matrix elements. Inserting complete sets of states in Eq. (8), one obtains the nucleon matrix element as well as additional matrix elements of higher energy states with the quantum numbers of the nucleon multiplied by overlap terms and time-dependent exponentials. For large enough time separations, the excited state contributions are suppressed compared to the nucleon ground state and one can then extract the desired matrix element. In order to increase the overlap with the nucleon state and decrease overlap with excited states we use Gaussian smeared quark fields $[16,17]$ for the construction of the interpolating fields:

$$
\begin{aligned}
q_{\text {smear }}^{a}(t, \mathbf{x}) & =\sum_{\mathbf{y}} F^{a b}(\mathbf{x}, \mathbf{y} ; U(t)) q^{b}(t, \mathbf{y}), \\
F & =(\mathbb{1}+\alpha H)^{n}, \\
H(\mathbf{x}, \mathbf{y} ; U(t)) & =\sum_{i=1}^{3}\left[U_{i}(x) \delta_{x, y-\hat{\imath}}+U_{i}^{\dagger}(x-\hat{\imath}) \delta_{x, y+\hat{\imath}}\right] .
\end{aligned}
$$

In addition, we apply Array Processor Experiment (APE) smearing to the gauge fields $U_{\mu}$ entering the hopping matrix $H$.

The Gaussian smearing parameters are optimized using the nucleon two-point function. We set $\alpha=0.2$ and $n=$ 125 [18]. The values are $\alpha=4.0$ and $n=50,70$, and 90 for $\beta=3.9,4.05$, and 4.2 respectively. For the APE smearing [19] we use 50 iteration steps and $\alpha_{\mathrm{APE}}=0.5$.

An optimized ratio [20-22] of the three-point function over a combination of two-point functions is used to cancel time-dependent exponentials and overlaps given by

$$
\begin{aligned}
& R_{\mu}\left(\Gamma_{\nu}, \vec{p}^{\prime}, \vec{p} ; t_{s}, t_{\mathrm{ins}}\right)=\frac{C_{\mu}\left(\Gamma_{\nu}, \vec{p}^{\prime}, \vec{p} ; t_{s}, t_{\mathrm{ins}}\right)}{C\left(\Gamma_{0}, \vec{p}^{\prime} ; t_{s}\right)} \\
& \quad \times \sqrt{\frac{C\left(\Gamma_{0}, \vec{p} ; t_{s}-t_{\mathrm{ins}}\right) C\left(\Gamma_{0}, \vec{p}^{\prime} ; t_{\mathrm{ins}}\right) C\left(\Gamma_{0}, \vec{p}^{\prime} ; t_{s}\right)}{C\left(\Gamma_{0}, \vec{p}^{\prime} ; t_{s}-t_{\mathrm{ins}}\right) C\left(\Gamma_{0}, \vec{p} ; t_{\mathrm{ins}}\right) C\left(\Gamma_{0}, \vec{p} ; t_{s}\right)}},
\end{aligned}
$$

where $t_{s}$ and $t_{\text {ins }}$ are taken to be relative to the source $t_{0}$ for simplicity. In the limit of large-time separations, $\left(t_{s}-t_{\text {ins }}\right) \gg 1$ and $t_{\text {ins }} \gg 1$, the lowest state dominates and the ratio becomes time independent

$$
R_{\mu}\left(\Gamma_{\nu} ; \vec{p}^{\prime}, \vec{p} ; t_{s} ; t_{\text {ins }}\right) \underset{t_{\text {ins }} \gg 1}{\stackrel{t_{s}-t_{\text {ins }} \gg 1}{\longrightarrow}} \Pi_{\mu}\left(\Gamma_{\nu} ; \vec{p}^{\prime}, \vec{p}\right) .
$$

$G_{E}\left(Q^{2}\right)$ and $G_{M}\left(Q^{2}\right)$ are extracted from linear combinations of $\Pi_{\mu}\left(\Gamma_{\nu} ; \vec{p}^{\prime}, \vec{p}\right)$ as expressed in Appendix A, with $Q^{2} \equiv-q^{2}$ the Euclidean momentum transfer squared.

Contracting the quark fields in Eq. (8) gives rise to two types of diagrams depicted in Fig. 1, namely the so-called connected and disconnected contributions. In the case of the connected diagram, the insertion operator couples to a valence quark and an all-to-all propagator arises between sink and insertion. We use sequential inversions through the sink that require keeping the sink-source time separation $t_{s}$, the projector, and the sink momentum $\vec{p}^{\prime}$ fixed. We perform additional sets of inversions to compute the three-point function for several values of $t_{s}$, for both the unpolarized and polarized projectors. We set $\vec{p}^{\prime}=\overrightarrow{0}$. We use an appropriately tuned multigrid algorithm [23-25] for the efficient inversion of the Dirac operator entering in the computation of the connected diagram. The disconnected diagram involves the disconnected quark loop correlated with the nucleon two-point correlator. The disconnected quark loop is given by
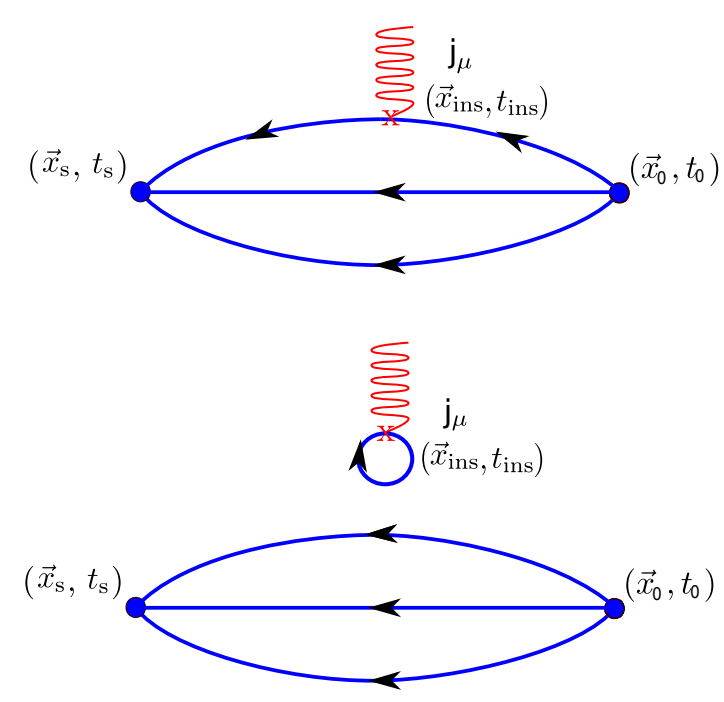

FIG. 1. Connected (upper panel) and disconnected (lower panel) contributions to the nucleon three-point function, with the source at $x_{0}$, the sink at $x_{s}$, and the current insertion $\left(j_{\mu}\right)$ at $x_{\text {ins }}$. 


$$
L\left(t_{\mathrm{ins}}, \vec{q}\right)=\sum_{\vec{x}_{\mathrm{ins}}} \operatorname{Tr}\left[D^{-1}\left(x_{\mathrm{ins}} ; x_{\mathrm{ins}}\right) \mathcal{G}\right] e^{i \vec{q} \cdot \vec{x}_{\mathrm{ins}}}
$$

where $D^{-1}\left(x_{\text {ins }} ; x_{\text {ins }}\right)$ is the quark propagator that starts and ends at the same point $x_{\text {ins }}$ and $\mathcal{G}$ is an appropriately chosen $\gamma$ structure. For the local vector current, which we use for the disconnected diagram, $\mathcal{G}=\gamma_{\mu}$. A direct computation of quark loops would need inversions from all spatial points on the lattice, making the evaluation unfeasible for our lattice size. We therefore employ stochastic techniques to estimate it combined with dilution schemes [26] that take into account the sparsity of the Dirac operator and its decay properties. Namely, in this work, we employ the hierarchical probing technique [10], which provides a partitioning scheme that eliminates contributions from neighboring points in the trace of Eq. (12) up to a certain coloring distance $2^{k}$. Using Hadamard vectors as the basis vectors for the partitioning, one needs $2^{d *(k-1)+1}$ vectors, where $d=4$ for a four-dimensional partitioning. Note that the computational resources required are proportional to the number of Hadamard vectors, and therefore in $d=4$ the dimensions increase 16-fold each time the probing distance $2^{k}$ doubles. Contributions entering from points beyond the probing distance are expected to be suppressed due to the exponential decay of the quark propagator and are treated with standard noise vectors which suppress all off-diagonal contributions by $1 / \sqrt{N_{r}}$, i.e.,

$$
\frac{1}{N_{r}} \sum_{r}\left|\xi_{r}\right\rangle\left\langle\xi_{r}\right|=1+\mathcal{O}\left(\frac{1}{\sqrt{N_{r}}}\right),
$$

where $N_{r}$ is the size of the stochastic ensemble. Hierarchical probing has been employed with great success in previous studies [27,28] for an ensemble with a pion mass of $317 \mathrm{MeV}$. For simulations at the physical point, it is expected that a larger probing distance is required since the light quark propagator decays more slowly at smaller quark masses. We avoid the need of increasing the distance by combining hierarchical probing with deflation of the low modes [11]. Namely, we construct the low mode contribution to the light quark loops by computing exactly the 200 smallest eigenvalues and corresponding eigenvectors of the squared Dirac operator and combine them with the contribution from the remaining modes, which are estimated using hierarchical probing. Additionally, we employ the one-end trick [29] used in our previous studies [30-33] and fully dilute in spin and color.

\section{B. Gauge ensembles and statistics}

For the extraction of the electromagnetic form factors we analyze one $N_{f}=2+1+1$ [5] and one $N_{f}=2$ ensemble. For both ensembles the quark masses are tuned to their physical values. The fermion action is the twisted mass fermion action with a clover term. Automatic $\mathcal{O}(a)$ improvement is achieved by tuning to maximal twist [34,35]. The $N_{f}=2+1+1 \mathrm{cB} 211.072 .64$ ensemble is simulated using a lattice of size $64^{3} \times 128$ with $L m_{\pi}=$ 3.62 [5], where $L$ is the spatial extent of the lattice. We determine the nucleon mass by fitting the effective mass in the large-time limit where the ground state dominates. The final value is chosen within a fit range where the value extracted is within half a standard deviation from the one determined by including in the fit the first excited state (two-state fit). The ratio of the nucleon to pion mass is $m_{N} / m_{\pi}=6.74(3)$ compared to the physical ratio of 6.8 . Therefore, we use directly the average proton and neutron mass of $0.9389 \mathrm{GeV}$ to set the scale. We find $a=$ $0.0801(4) \mathrm{fm}$. For the pion mass we find $m_{\pi}=$ $0.1393(7) \mathrm{MeV}$ consistent with the average physical pion mass. Our current value of the lattice spacing is an update compared to the one given in Ref. [5] using higher statistics where the values are consistent.

To assess finite volume effects, we use two $N_{f}=2$ ensembles, which only differ in their volume; namely one has $L m_{\pi}=2.98$ and the other $L m_{\pi}=3.97$. We will refer to them as the cA2.09.48 and cA2.09.64 ensembles, respectively. We note that since the pion mass is not exactly at the physical value we interpolate to the physical pion mass using one-loop chiral perturbation theory. We include a systematic error on the extracted lattice spacing determined as the difference in the mean value obtained using one-loop chiral perturbation theory and heavy baryon chiral perturbation theory. This systematic error on the lattice spacing appears for the two $N_{f}=2$ ensembles, while it is absent in the case of the cB211.072.64 ensemble. Results on the form factors for the $N_{f}=2$ ensemble with $L m_{\pi}=2.98$ are from Ref. [7] while results for the other two ensembles are reported here for the first time. The simulation parameters of all three ensembles considered in this work are tabulated in Table I.

For the analysis of the cB211.072.64 ensemble we use 750 configurations separated by four trajectories. For the connected contributions we evaluate the three-point function for five sink-source time separations in the range 0.96 to $1.60 \mathrm{fm}$ increasing the number of source positions per configuration as we increase the time separation so as to keep the statistical error approximately constant. In Table II we give the statistics used in the calculation of the connected three-point functions.

For the evaluation of the disconnected contributions we use $N_{\text {srcs }}=200$ source positions to generate the nucleon two-point functions that are correlated with the quark loop to produce the disconnected contribution to the three-point function. We find that the volume is sufficiently large so that the data extracted from this large number of randomly distributed source positions on the same configuration are statistically independent. Nevertheless, we average over all source positions for each configuration and take the averaged correlation function as 
TABLE I. Simulation parameters for the $N_{f}=2+1+1$ [5] and $N_{f}=2$ [36] ensembles used in this work. When two errors are given, the first error is statistical and the second is systematic. The systematic error in the lattice spacing of the $N_{f}=2$ ensembles is obtained as described in the text, while the systematic error in the pion mass provided in physical units is propagated from the lattice spacing.

\begin{tabular}{|c|c|c|c|c|c|c|c|c|c|c|c|}
\hline Ensemble & $c_{\mathrm{SW}}$ & $\beta$ & $N_{f}$ & $a(\mathrm{fm})$ & Vol. & $a m_{\pi}$ & $m_{\pi} L$ & $a m_{N}$ & $m_{N} / m_{\pi}$ & $m_{\pi}(\mathrm{GeV})$ & $L(\mathrm{fm})$ \\
\hline cB211.072.64 & 1.69 & 1.778 & $2+1+1$ & $0.0801(4)$ & $64^{3} \times 128$ & $0.05658(6)$ & 3.62 & $0.3813(19)$ & $6.74(3)$ & $0.1393(7)$ & $5.12(3)$ \\
\hline cA2.09.64 & 1.57551 & 2.1 & 2 & $0.0938(3)(1)$ & $48^{3} \times 96$ & $0.06208(2)$ & 2.98 & $0.4436(11)$ & $7.15(2)$ & $0.1306(4)(2)$ & $4.50(1)$ \\
\hline cA2.09.48 & 1.57551 & 2.1 & 2 & $0.0938(3)(1)$ & $64^{3} \times 128$ & $0.06193(7)$ & 3.97 & $0.4421(25)$ & $7.14(4)$ & $0.1303(4)(2)$ & $6.00(2)$ \\
\hline
\end{tabular}

TABLE II. Statistics for the evaluation of the connected threepoint functions for the $N_{f}=2+1+1 \mathrm{cB} 211.072 .64$ ensemble. Columns from left to right are the sink-source time separations, the number of configurations analyzed, the number of source positions per configuration chosen randomly, and the total number of measurements for each time separation.

\begin{tabular}{lccr}
\hline \hline$t_{s} / a$ & $N_{\text {cnfs }}$ & $N_{\text {srcs }}$ & $N_{\text {meas }}$ \\
\hline 12 & 750 & 4 & 3000 \\
14 & 750 & 6 & 4500 \\
16 & 750 & 16 & 12000 \\
18 & 750 & 48 & 36000 \\
20 & 750 & 64 & 48000 \\
\hline \hline
\end{tabular}

one statistic in our jackknife error analysis. As mentioned in the previous section, for the evaluation of the light quark loops we use the first 200 low modes of the squared Dirac operator to reconstruct exactly part of the loop. The contribution from the high modes is estimated stochastically using one noise vector per configuration combining hierarchical probing, one-end trick, and spin-color dilution. For the hierarchical probing we use distance eight coloring resulting in 512 Hadamard vectors, which when combined with spin-color dilution leads to 6144 inversions per configuration. We note that the next coloring distance would demand 8192 Hadamard vectors, resulting in 98304 inversions per configuration after combining with spin-color dilution, making such a computation more than an order of magnitude more expensive.

TABLE III. Details on the setup for the evaluation of the light disconnected diagrams. $N_{\mathrm{cnfs}}$ is the number of configurations analyzed, $N_{\text {def }}$ is the number of low modes we deflate, $N_{r}$ the number of noise vectors, and $N_{\mathrm{Had}}$ the number of Hadamard vectors. $N_{\text {sc }}$ corresponds to spin-color dilution and $N_{\text {inv }} /$ conf is the total number of inversions per configuration. $N_{\text {srcs }}$ is the number of randomly distributed smeared point sources per configuration used to obtain the nucleon two-point functions and $N_{\text {meas }}$ the total number of measurements.

\begin{tabular}{lcccccccccc}
\hline \hline \multicolumn{4}{c}{ Loops } & & \multicolumn{3}{c}{ Two point } \\
${$\cline { 1 - 2 }$} }$ & $N_{\text {cnfs }}$ & $N_{\text {def }}$ & $N_{r}$ & $N_{\text {Had }}$ & $N_{\text {sc }}$ & $N_{\text {inv }} /$ conf & $N_{\text {srcs }}$ & $N_{\text {meas }}$ \\
\hline cB211.072.64 & 750 & 200 & 1 & 512 & 12 & 6144 & 200 & 150000 \\
cA2.09.48 & 2120 & $\ldots$ & 2250 & $\ldots$ & $\ldots$ & 2250 & 100 & 212000 \\
\hline \hline
\end{tabular}

For the computation of the disconnected contributions for the $N_{f}=2$ cA2.09.48 ensemble computed previously we used only the one-end trick and 2250 noise vectors for the calculation. Two-point functions were computed for 100 source positions. More details can be found in Ref. [6]. In Table III we summarize the parameters for the computation of the disconnected three-point functions.

The cA2.09.64 ensemble is used to check for finite volume effects, comparing the connected contributions to those of the cA2.09.48 ensemble. For the latter, the setup is reported in Ref. [7] and summarized in Table I. For the larger lattice size ensemble, we analyze three sink-source time separations in the range of 1.1 to $1.5 \mathrm{fm}$. We fix the number of source positions per configuration to 16 and we use more configurations for the larger time separations to control statistical error. In Table IV we summarize the statistics for both $N_{f}=2$ ensembles.

\section{Excited states contamination}

Assessment of excited state effects is imperative for the proper extraction of the desired nucleon matrix element. However, ensuring ground state dominance is a delicate process due to the exponentially increasing statistical noise with increasing sink-source separation. We use four methods to study the effect of excited states and identify the final results based on a critical comparison among these methods. Only by employing these different methods can one

TABLE IV. Statistics for the evaluation of the connected threepoint functions for the cA2.09.64 and cA2.09.48 ensembles. For the latter, for $t_{s} / a=16,18$ the connected three-point functions have been computed only for the unpolarized projector. The notation is as in Table II.

\begin{tabular}{lcrr}
\hline \hline$t_{s} / a$ & $N_{\mathrm{cnfs}}$ & $N_{\text {srcs }}$ & $N_{\text {meas }}$ \\
\hline $\mathrm{cA} 2.09 .64: N_{f}=2$ & $64^{3} \times 128$ & ensemble & \\
\hline 12 & 333 & 16 & 5328 \\
14 & 515 & 16 & 8240 \\
16 & 1040 & 16 & 16640 \\
\hline $\mathrm{cA} 2.09 .48: N_{f}=2$ & $48^{3} \times 96$ ensemble & & \\
\hline $10,12,14$ & 578 & 16 & 9248 \\
16 & 530 & 88 & 46640 \\
18 & 725 & 88 & 63800 \\
\hline \hline
\end{tabular}


reach a reliable assessment of excited state contributions and extract the nucleon matrix element of interest. The methods employed are as follows:

Plateau method: In this method we use the ratio in Eq. (10) and identify a time-independent window (plateau) as we increase $t_{s}$. The converged plateau value then yields the desired matrix element.

Two-state method: Within this method we fit the twoand three-point functions considering contributions up to the first excited state using the expressions

$$
\begin{aligned}
& C\left(\vec{p}, t_{s}\right)=c_{0}(\vec{p}) e^{-E_{0}(\vec{p}) t_{s}}+c_{1}(\vec{p}) e^{-E_{1}(\vec{p}) t_{s}}, \\
& C_{\mu}\left(\Gamma_{\nu}, \vec{p}^{\prime}, \vec{p}, t_{s}, t_{\text {ins }}\right) \\
& =A_{00}^{\mu}\left(\Gamma_{\nu}, \vec{p}^{\prime}, \vec{p}\right) e^{-E_{0}\left(\vec{p}^{\prime}\right)\left(t_{s}-t_{\text {ins }}\right)-E_{0}(\vec{p}) t_{\text {ins }}} \\
& \quad+A_{01}^{\mu}\left(\Gamma_{\nu}, \vec{p}^{\prime}, \vec{p}\right) e^{-E_{0}\left(\vec{p}^{\prime}\right)\left(t_{s}-t_{\text {ins }}\right)-E_{1}(\vec{p}) t_{\text {ins }}} \\
& \quad+A_{10}^{\mu}\left(\Gamma_{\nu}, \vec{p}^{\prime}, \vec{p}\right) e^{-E_{1}\left(\vec{p}^{\prime}\right)\left(t_{s}-t_{\text {ins }}\right)-E_{0}(\vec{p}) t_{\text {ins }}} \\
& \quad+A_{11}^{\mu}\left(\Gamma_{\nu}, \vec{p}^{\prime}, \vec{p}\right) e^{-E_{1}\left(\vec{p}^{\prime}\right)\left(t_{s}-t_{\text {ins }}\right)-E_{1}(\vec{p}) t_{\text {ins }}} .
\end{aligned}
$$

In Eqs. (14) and (15) $E_{0}(\vec{p})$ and $E_{1}(\vec{p})$ are the energies of the ground and first excited states with total momentum $\vec{p}$, respectively. The ground state corresponds to a single particle state and therefore one can use the continuum dispersion relation, $E_{0}(\vec{p})=\sqrt{\vec{p}^{2}+m_{N}^{2}}$, with $\vec{p}=\frac{2 \pi}{L} \vec{n}$ with $\vec{n}$ a lattice vector with components $n_{i} \in\left(-\frac{L}{2 a}, \frac{L}{2 a}\right]$. The continuum dispersion relation is satisfied for all $Q^{2}$ values considered in this work. The first excited state, on the other hand, can be a two-particle state. We, thus, fit simultaneously the two-point functions with momenta $\vec{p}$ and $\vec{p}^{\prime}$ and the three-point function involving in total 11 parameters. Note that for nonzero momentum transfer, $A_{01}^{\mu}\left(\Gamma_{\nu}, \vec{p}^{\prime}, \vec{p}\right) \neq A_{10}^{\mu}\left(\Gamma_{\nu}, \vec{p}^{\prime}, \vec{p}\right)$. This allows us to extract the matrix element given by

$$
\Pi_{\mu}\left(\Gamma_{\nu} ; \vec{p}^{\prime}, \vec{p}\right)=\frac{A_{00}^{\mu}\left(\Gamma_{\nu}, \vec{p}^{\prime}, \vec{p}\right)}{\sqrt{c_{0}\left(\vec{p}^{\prime}\right) c_{0}(\vec{p})}} .
$$

Summation method: Summing over $t_{\text {ins }}$ in the ratio of Eq. (10) yields a geometric sum [37,38] from which we obtain

$$
\begin{aligned}
R_{\mu}^{\mathrm{sum}}\left(\Gamma_{\nu} ; \vec{p}^{\prime}, \vec{p} ; t_{s}\right) & =\sum_{t_{\mathrm{ins}}=a}^{t_{s}-a} R_{\mu}\left(\Gamma_{\nu} ; \vec{p}^{\prime}, \vec{p} ; t_{s} ; t_{\mathrm{ins}}\right) \\
& =c+\Pi_{\mu}\left(\Gamma_{\nu} ; \vec{p}^{\prime}, \vec{p}\right) \times t_{s}+\cdots
\end{aligned}
$$

where the ground state contribution, $\Pi_{\mu}\left(\Gamma_{\nu} ; \vec{p}^{\prime}, \vec{p}\right)$, is extracted from the slope of a linear fit with respect to $t_{s}$. The sink-source time separation $t_{s}$ considered in the fit should be large enough to suppress higher order contributions.
Derivative summation method: Instead of performing a linear fit in Eq. (17) to extract the matrix element, one can take finite differences to the summed ratio [39] as follows:

$$
\begin{aligned}
& d R_{\mu}^{\operatorname{sum}}\left(\Gamma_{\nu} ; \vec{p}^{\prime}, \vec{p} ; t_{s}\right) \\
& =\frac{R_{\mu}^{\operatorname{sum}}\left(\Gamma_{\nu} ; \vec{p}^{\prime}, \vec{p} ; t_{s}+d t_{s}\right)-R_{\mu}^{\mathrm{sum}}\left(\Gamma_{\nu} ; \vec{p}^{\prime}, \vec{p} ; t_{s}\right)}{d t_{s}}
\end{aligned}
$$

and fit to a constant to extract the desired matrix element.

\section{ANALYSIS OF LATTICE RESULTS}

\section{A. Isovector and connected isoscalar form factors}

The isovector combination gives the difference between the proton and neutron form factors, and in this case, only the connected diagram contributes since disconnected contributions cancel, up to cutoff effects of $\mathcal{O}\left(a^{2}\right)$. For the connected diagram we use a frame where the nucleon final momentum is zero, thus $\vec{q}=-\vec{p}$. In Figs. 2 and 3 we show the ratios defined in Eq. (10) as a function of the sink-source time separation, and for three values of the momentum transfer squared, that is for $Q^{2}=0.057 \mathrm{GeV}^{2}$, $Q^{2}=0.219 \mathrm{GeV}^{2}$, and $Q^{2}=0.554 \mathrm{GeV}^{2}$. In a frame where the final momentum of the nucleon $\vec{p}^{\prime}$ is zero, the expressions in Appendix A given by Eqs. (A1) and (A2) reduce to Eqs. (A4)-(A6), giving separately the electric and magnetic form factors. We note that for the electric form

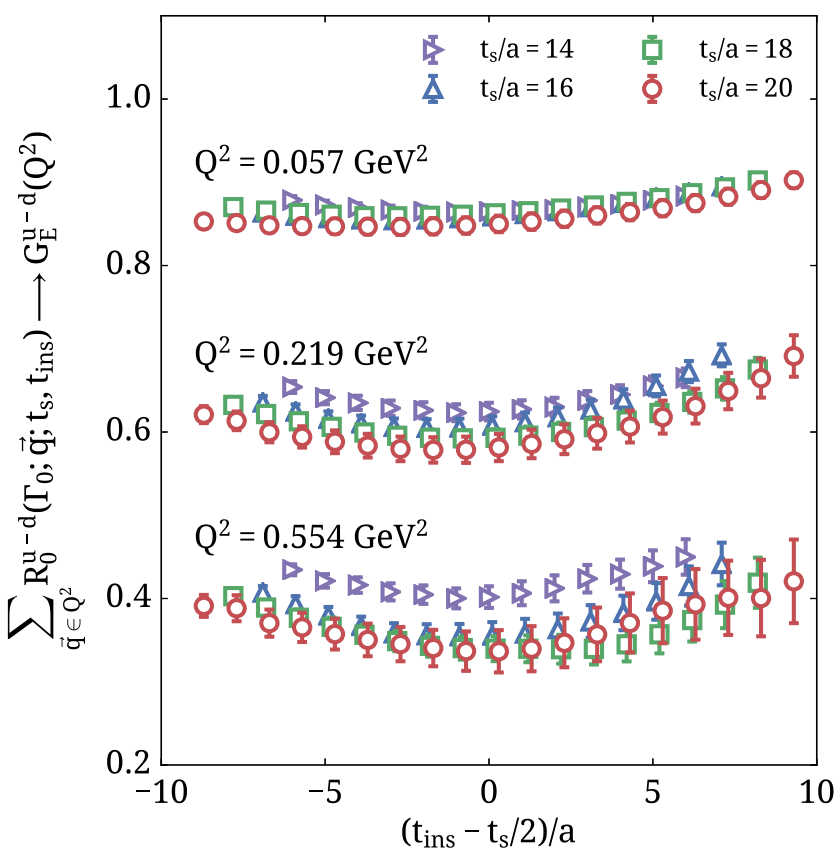

FIG. 2. Ratio yielding the isovector electric form factor. The sink-source time separations are $t_{s} / a=14$ (right triangles), $t_{s} / a=16$ (triangles), $t_{s} / a=18$ (squares), and $t_{s} / a=20$ (circles). We present the ratio for three $Q^{2}$ values, namely $0.057,0.219$, and $0.554 \mathrm{GeV}^{2}$, from top to bottom. 


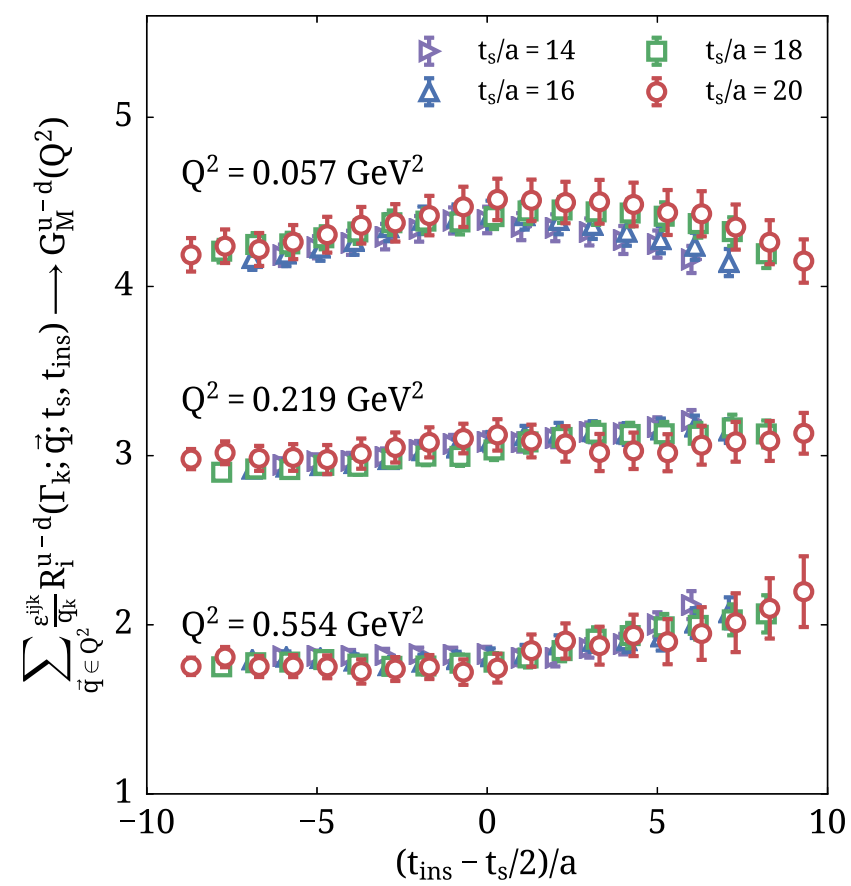

FIG. 3. Ratio yielding the isovector magnetic form factor. The notation is the same as in Fig. 2. factor Eq. (A4) leads to much more precise results compared to Eq. (A5) and therefore we use only Eq. (A4). In the case of the ratio determining $G_{E}^{u-d}\left(Q^{2}\right)$, as $t_{s}$ increases, the plateau value decreases with larger deviations as $Q^{2}$ increases. This shows that at larger $Q^{2}$ values contamination due to excited states is more severe. In the case of $G_{M}\left(Q^{2}\right)$, excited states are suppressed and only a small variation with $t_{s}$ is observed.

We further investigate effects due to excited states by employing the summation and two-state fits. In Fig. 4 we show linear fits to the summed ratio for three different values of $Q^{2}$. The slope gives the nucleon matrix element. All three momenta follow well the linear behavior, within the statistical error, indicating that contributions from higher order terms are suppressed. In the right panel of Fig. 4, we demonstrate the plateaus for the derivative summation method, fitting to a constant to extract the matrix element of the ground state. Within statistical accuracy all three momenta are indeed flat, and are thus described well by a constant.

In Fig. 5 we show the results extracted using two-state fits for both electric and magnetic form factors. The data correspond to the ratio of Eq. (10) and the curves are obtained by fitting simultaneously the three- and two-point

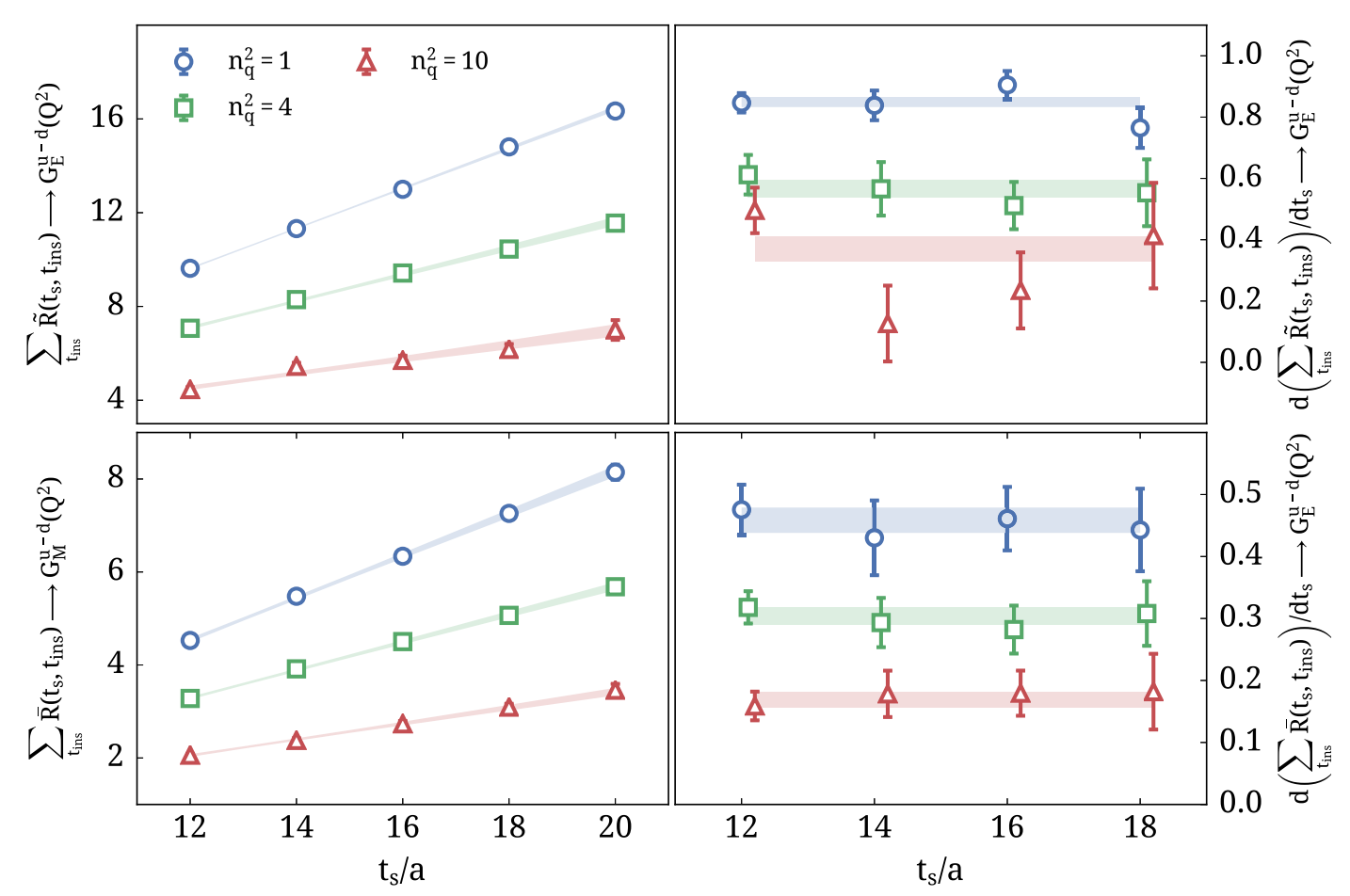

FIG. 4. We define $\tilde{R}\left(t_{s}, t_{\text {ins }}\right) \equiv \sum_{\vec{q} \in Q^{2}} R_{0}^{u-d}\left(\Gamma_{0} ; \vec{q} ; t_{s}, t_{\text {ins }}\right)$ and $\bar{R}\left(t_{s}, t_{\text {ins }}\right) \equiv \sum_{\vec{q} \in Q^{2}} \frac{\epsilon^{i j k}}{q_{k}} R_{i}^{u-d}\left(\Gamma_{k} ; \vec{q} ; t_{s}, t_{\text {ins }}\right)$. Left panel: The summed ratio of Eq. (17) as a function of the sink-source time separation for three momenta, namely $n_{q}^{2}=1$ (blue circles), $n_{q}^{2}=4$ (green squares), and $n_{q}^{2}=10$ (red triangles) corresponding to $Q^{2}=0.057,0.219$, and $0.554 \mathrm{GeV}^{2}$ for the isovector electric (top) and isovector magnetic (bottom) form factors. The bands are fits to a linear form. Right panel: The derivative of the summed ratio as in Eq. (18), using the same notation as that of the left panel. The bands are fits to a constant. 


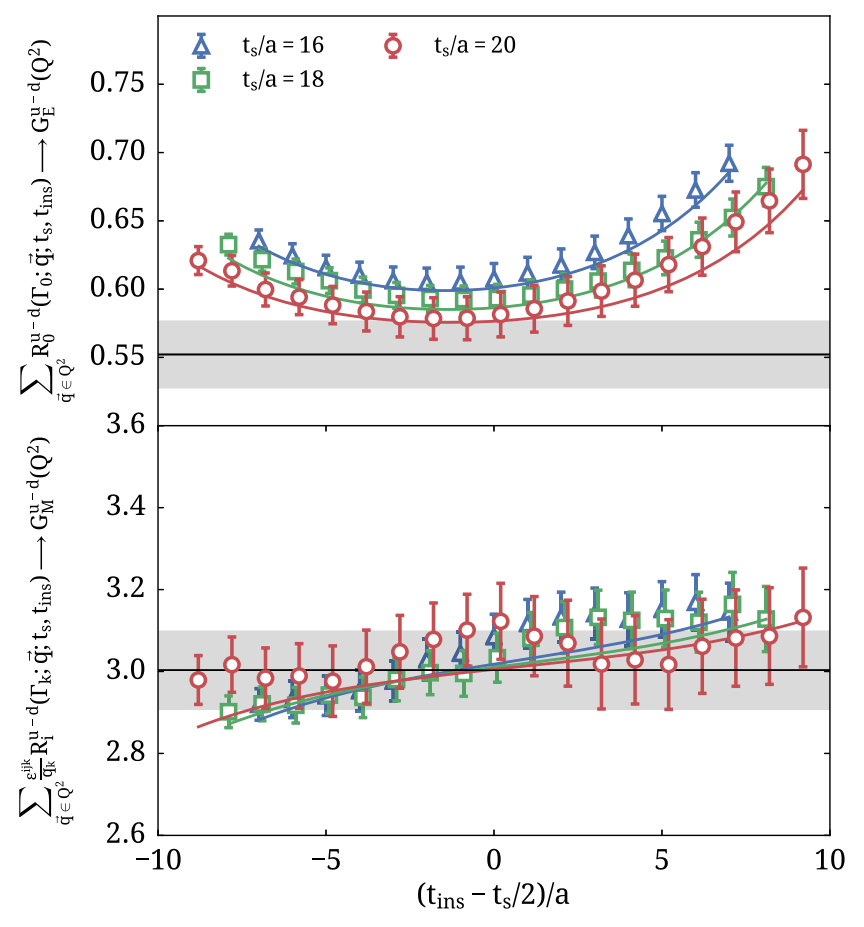

FIG. 5. Ratio yielding the isovector electric (top) and the isovector magnetic (bottom) form factors, for the three largest sink-source separations, following the notation of Fig. 2. The curves show the result of the two-state fit method, while the gray horizontal band is the extracted value of the nucleon matrix elements and its error. We show the case for $Q^{2}=0.219 \mathrm{GeV}^{2}$.

functions to Eqs. (15) and (14). The gray horizontal band shows the nucleon matrix element value and error extracted from the two-state fit as in Eq. (16). For the electric form factor, the ratio shows a trend towards lower values as we increase the sink-source separation, with $t_{s} / a=20$ becoming compatible with the value extracted from the two-state fit. In the case of the magnetic form factor, the value extracted from the two-state fit is compatible with the ratio for all time separations considered confirming the weak dependence of the matrix element on the sink-source time separation observed in the plateau method.

In Fig. 6 we show the extracted values for the matrix element yielding the isovector electromagnetic form factors. We compare the plateau, summation, derivative summation, and two-state fit methods. For the plateau method we show the value extracted from the constant fit for all sink-source separations available. For the other cases we vary the lower fit range, keeping the upper range fixed to $t_{s} / a=20$. We seek the earliest agreement between the plateau method and the other three cases. As already pointed out, the isovector electric form factor shows more severe excited state effects for large $Q^{2}$ values and we therefore take the largest time separation for the plateau method to fulfill our criterion for agreement with the other methods. For the isovector magnetic form factor, although excited state effects are mild, we still observe a shift to larger values for the smallest $Q^{2}$ and, therefore, we conservatively use the largest time separation available also in this case. An additional observation is that the summation and derivative summation methods produce compatible results with similar accuracy, as can be seen in Fig. 6, and thus from now on we will restrict to showing results only from the summation method.

In Fig. 7, we present our results for $G_{E}^{u-d}\left(Q^{2}\right)$ and $G_{M}^{u-d}\left(Q^{2}\right)$ as a function of the momentum transfer squared $Q^{2}$. We limit the plot up to $Q^{2}=0.5 \mathrm{GeV}^{2}$ to make visible the values extracted using the plateau at the four largest separations, the summation, and the two-state fit approaches. For the summation and two-state fit we show the values indicated by the filled symbols in Fig. 6. As can be seen, for the electric form factor the effects of excited states are small for small values of $Q^{2}$ but become more severe for higher $Q^{2}$ values, with the extracted value decreasing with increasing time separation in line with the observation made in Fig. 2. For $G_{M}^{u-d}\left(Q^{2}\right)$, excited state effects are small for larger $Q^{2}$ values whereas for smaller $Q^{2}$ values there is a systematic increase in the values of the form factor with the time separation.

For the extraction of the connected isoscalar form factors we follow a similar analysis procedure as in the isovector case. In Fig. 8 we present the connected contribution to isoscalar electric and magnetic form factors comparing the plateau, summation, and two-state fit methods. Excited states have a smaller effect on the isoscalar form factors being detectable only for the magnetic form factor at small values of $Q^{2}$ where the two-state fit yields systematically larger values. Given that there is agreement between the plateau values for the largest time separation and the twostate fit we will use the plateau value as the final result for the form factors. The deviation from the values determined from the two-state fits are then taken as an estimate of the systematic error due to excited states. Since we will be using the plateau values in the case of the disconnected contribution since two-state fits are not stable in that case we do the same for the connected contribution for consistency.

\section{B. Disconnected contributions}

A major component of this work is the evaluation of the disconnected contributions shown diagrammatically in Fig. 1 that enter in the evaluation of the isoscalar as well as in the proton and neutron form factors.

The disconnected quark loops are computed using the formalism described in Sec. III B with the statistics summarized in Table III. As already discussed, the hierarchical probing method combined with deflation of the low eigenmodes provides an accurate determination of the diagonal of the quark propagator entering in the evaluation of the quark loops. It is thus preferable to use the local vector current for the evaluation of the disconnected 


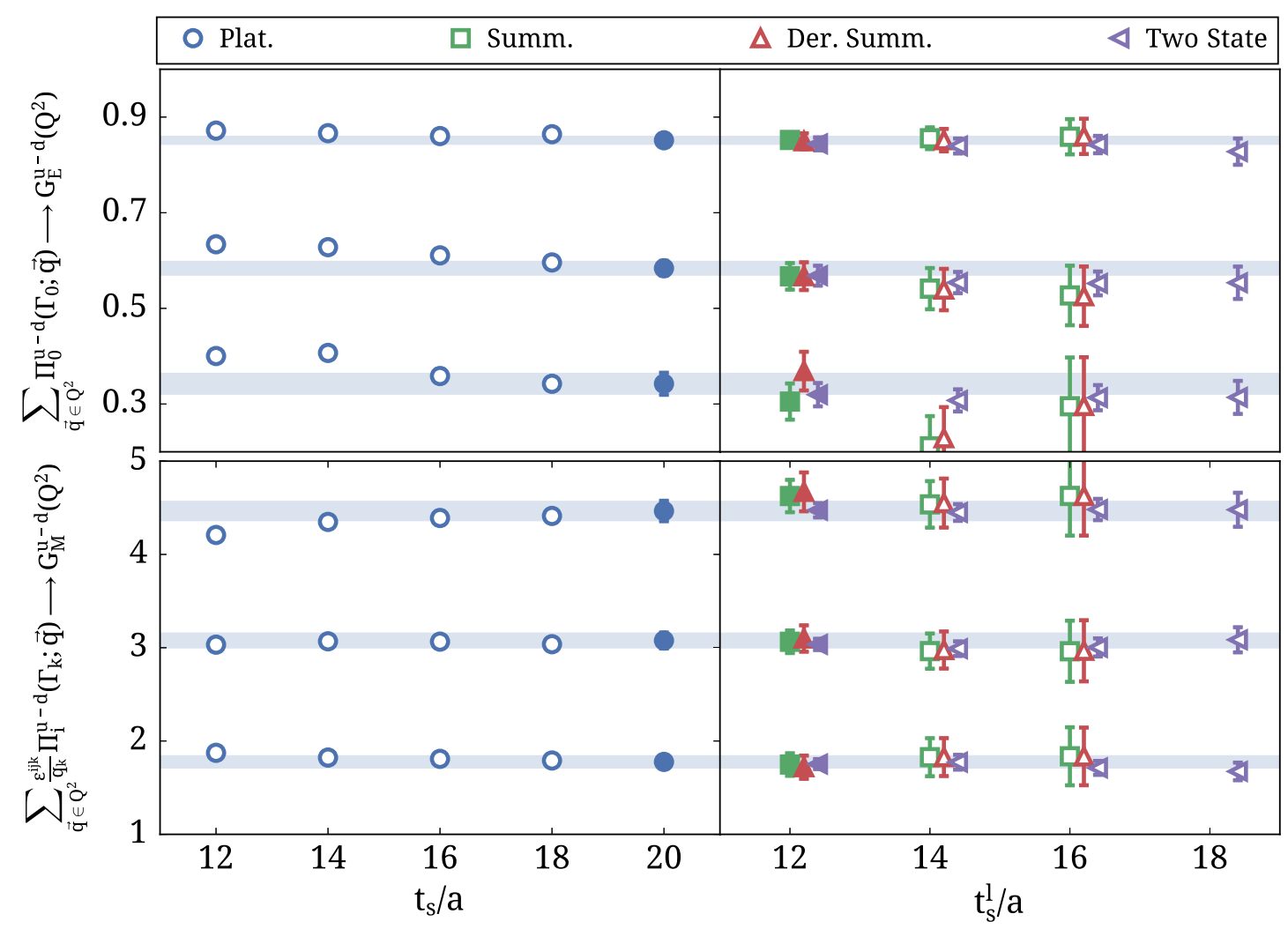

FIG. 6. Results for the matrix element yielding $G_{E}^{u-d}$ (upper panel) and $G_{M}^{u-d}$ (lower panel), for $Q^{2}=0.057 \mathrm{GeV}, Q^{2}=0.219 \mathrm{GeV}$, and $Q^{2}=0.554 \mathrm{GeV}^{2}$ from top to bottom. In the left column we show the extracted values using the plateau method (blue circles) for all five separations analyzed, while in the right panel we show the values extracted using the summation (green squares), derivative summation (red upper triangles), and two-state fit (purple left triangles) approaches as we change the lower fit range ( $\left.\mathrm{t}_{\mathrm{s}}^{1} / \mathrm{a}\right) \mathrm{keeping}$ the upper fit range fixed to $t_{s} / a=20$. The filled circle and band show the value and statistical error used to quote our final result, while the other filled symbols show the fit ranges for the two-state fit and summation methods that will be used in the figures that follow.

contributions since the conserved current includes nondiagonal terms. We therefore need the renormalization function $Z_{V}$, which is determined nonperturbatively, in the $\mathrm{RI}^{\prime}$-MOM scheme, employing momentum sources. We perform a perturbative subtraction of $\mathcal{O}\left(g^{2} a^{\infty}\right)$ terms, as described in Refs. [40,41], which subtracts the leading cutoff effects leaving only a weak dependence on the renormalization scale $(a \mu)^{2}$, as shown in Fig. 9. We find a value of $Z_{V}=0.728(1)$ where the error is statistical. Alternatively, $Z_{V}$ can be determined at $Q^{2}=0$, by taking the ratio of $G_{E}^{u-d}(0)$ computed with the local current to $G_{E}^{u-d}(0)$ computed using the lattice conserved current. This ratio yields a value of $0.715(3)$. Although this is $2 \%$ smaller than $Z_{V}$ as determined from the vertex function, the difference between them is still an order of magnitude smaller as compared to the statistical errors for the disconnected contributions. In what follows we use $Z_{V}=$ 0.728 (1) to renormalize the matrix elements computed using the local current, since this determination has taken into account higher order cutoff effects as compared to the one determined from the ratio. We note that $Z_{V}$ only enters in the disconnected three-point function. A more detailed description of the renormalization procedure including other renormalization functions will be provided in a future publication.

Disconnected quark loops are evaluated for every time slice allowing us to compute the three-point function for every combination of $t_{s}$ and $t_{\text {ins. }}$. As in the case of the connected contributions, we are seeking a reasonable window in $t_{s}$ to extract the nucleon matrix elements, where excited states are sufficiently suppressed and noise is not prohibitively large. In contrast to the connected diagram, where we have results only for the case $\vec{p}^{\prime}=\overrightarrow{0}$, for the disconnected diagrams we have all sink momenta at no additional cost. We analyze, besides $\vec{p}^{\prime}=\overrightarrow{0}$, the matrix element for the six final momenta with $\vec{p}^{\prime}=$ $\pm \frac{2 \pi}{L} \hat{n}$, with $\hat{n}=\hat{x}, \hat{y}$, or $\hat{z}$, i.e., the unit vector in one of the three spatial directions. Given that the statistical errors in the case of the disconnected diagrams are larger as compared to the connected diagrams, we restrict ourselves in using the plateau method for different values of $t_{s}$ in order to check for ground state dominance. This is because the two-state fits are problematic given the larger errors of the disconnected diagrams. 

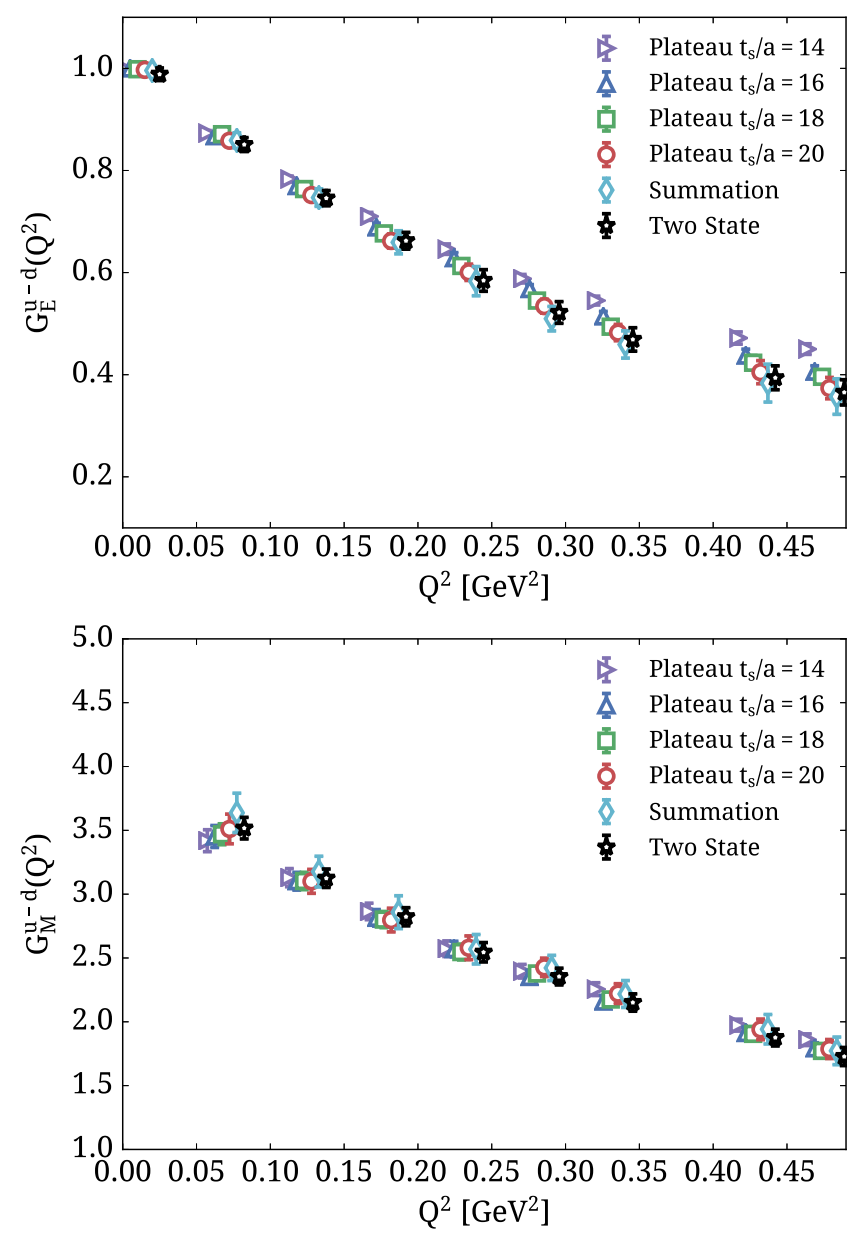

FIG. 7. The isovector electric (upper panel) and magnetic (lower panel) form factors as a function of $Q^{2}$. We show the values extracted from fitting the plateau for the four largest $t_{s}$ values, namely $t_{s} / a=14$ (right triangles), $t_{s} / a=16$ (triangles), $t_{s} / a=18$ (squares), and $t_{s} / a=20$ (circles), compared to the summation method (diamonds) and using two-state fits (stars). Results from different methods are slightly shifted to the right for clarity.

Whenever they work they yield large errors and are consistent with the plateau extraction.

In Fig. 10 we present our results for the disconnected contributions to $G_{E}^{u+d}\left(Q^{2}\right)$ and $G_{M}^{u+d}\left(Q^{2}\right)$ up to $Q^{2}=$ $1 \mathrm{GeV}^{2}$ for three time separations, $t_{s}=0.96,1.12$, and $1.28 \mathrm{fm}$. We can achieve a relative statistical error that is less than $20 \%$ for up to $t_{s}=14 a \sim 1.12 \mathrm{fm}$, which is unprecedented given that we are using a physical pion mass ensemble. As we increase the time separation from $t_{s} / a=$ 12 to $t_{s} / a=14$ we observe, for both $G_{E}^{u+d}\left(Q^{2}\right)$ and $G_{M}^{u+d}\left(Q^{2}\right)$, that there is a trend for larger values, while the results extracted for $t_{s} / a=16$ are in a very good agreement with those extracted for $t_{s} / a=14$ for most $Q^{2}$ values, albeit with larger errors. We, therefore, take as our final result for the disconnected contribution the value extracted using $t_{s} / a=14$ for both $G_{E}^{u+d}\left(Q^{2}\right)$ and
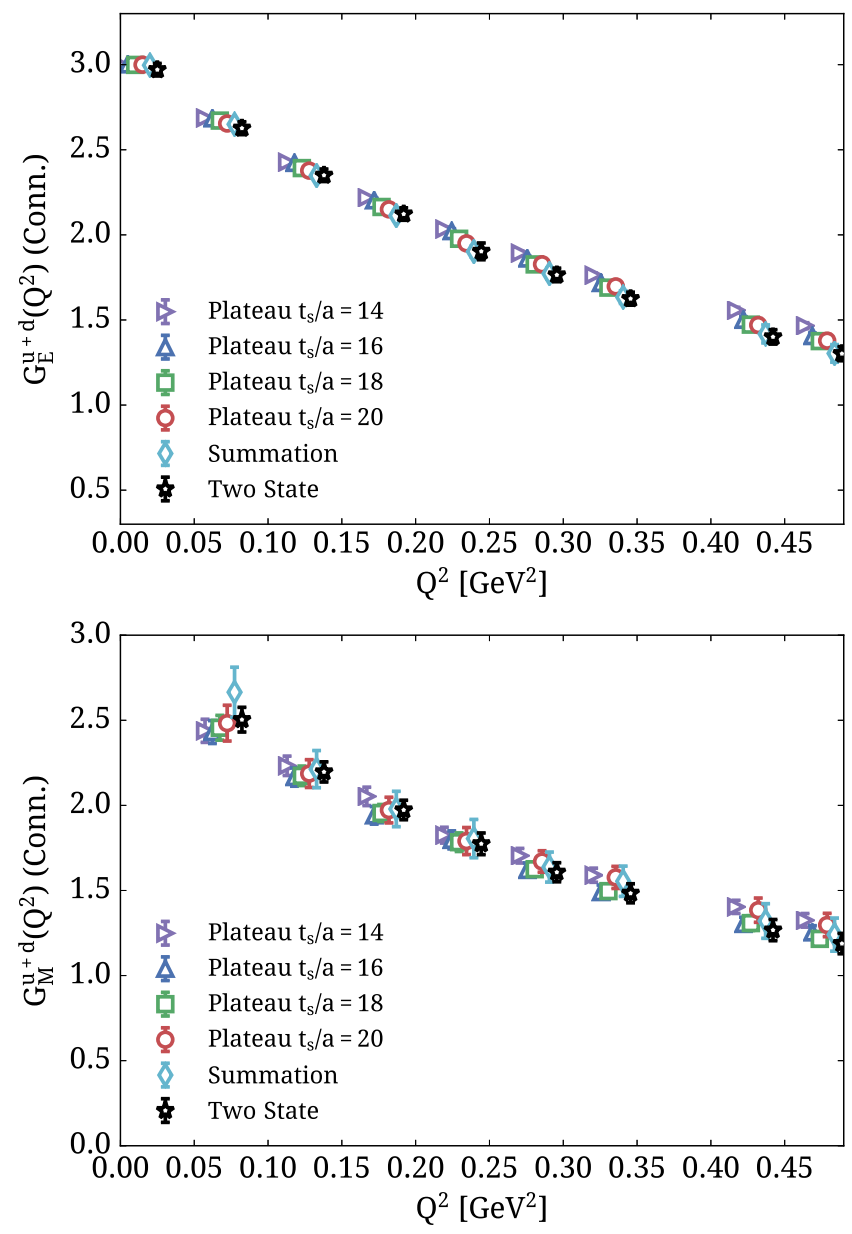

FIG. 8. Connected contribution to the isoscalar electric (upper panel) and magnetic (lower panel) form factors. The notation is the same as in Fig. 7.

$G_{M}^{u+d}\left(Q^{2}\right)$. We use the difference between the central value of the results at $t_{s}=14 a$ and $t_{s}=16 a$ as an estimate of the systematic error from excited state effects when we quote quantities that include disconnected contributions.

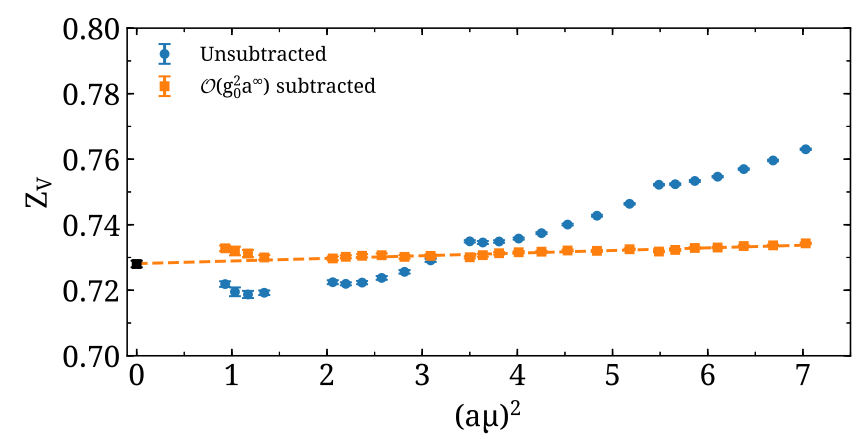

FIG. 9. The renormalization constant $Z_{V}$ as a function of the renormalization scale squared $(a \mu)^{2}$ before (blue circles) and after (orange squares) performing the subtraction of the $\mathcal{O}\left(g^{2} a^{\infty}\right)$ terms. The dashed line is a linear fit to the latter and the point at $(a \mu)^{2}=0$ (black square) is the result of the fit. 

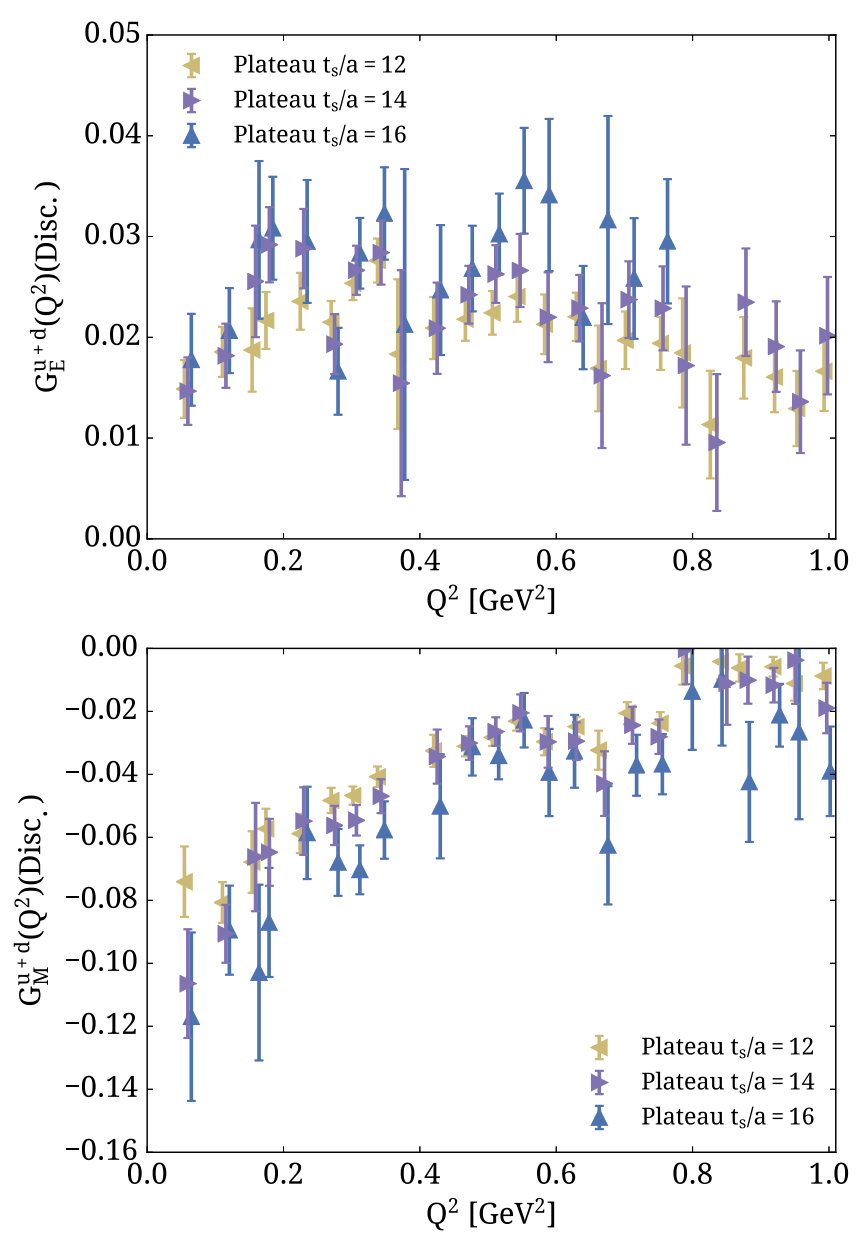

FIG. 10. The disconnected contributions to $G_{E}^{u+d}$ (top) and $G_{M}^{u+d}$ (bottom) using the plateau method for $t_{s} / a=12$ (left triangles), $t_{s} / a=14$ (right triangles), and $t_{s} / a=16$ (upright triangles). Points from closely spaced $Q^{2}$ have been averaged for demonstration, and results from different time separations have been slightly shifted to the right for clarity.

\section{ASSESSMENT OF LATTICE ARTIFACTS}

We collectively discuss here lattice artifacts that may lead to systematic errors. Since we use simulations with physical values of the light quark masses no chiral extrapolation is needed eliminating an uncontrolled uncertainty present in past lattice QCD computations of these quantities.

(i) Disconnected contributions: The main novelty of this work is the accurate computation of the light quark disconnected contributions using simulations with quark masses tuned to their physical values. This enables us, for the first time, to eliminate this systematic uncertainty in the determination of the proton and neutron form factors at the physical point. Strange quark loops contribution is not included in this study, but we know from previous studies $[6,27,42]$ that it is
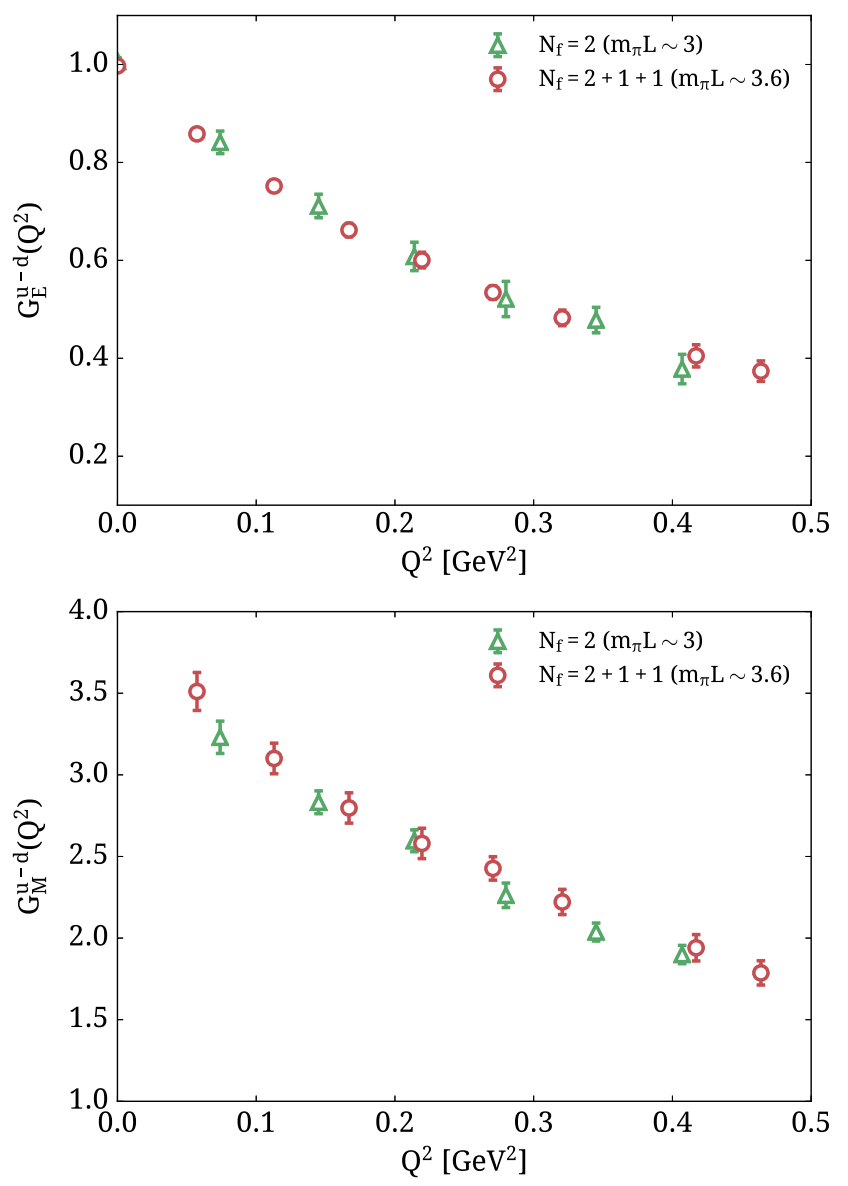

FIG. 11. Comparison of the isovector electric (top panel) and magnetic (lower panel) form factors between the $N_{f}=2$ cA2.09.48 ensemble [7] (green triangles) and the $N_{f}=2+1+1$ cB211.072.64 ensemble (red circles). Results are extracted using the plateau method for sink-source time separation $t_{s} \simeq 1.3 \mathrm{fm}$.

much smaller compared to the statistical error of the connected contribution.

(ii) Quenching effects: The analysis of the $N_{f}=2+$ $1+1$ ensemble and two $N_{f}=2$ ensembles allows us to check for unquenching effects due to the strange and charm quarks. In Figs. 11 and 12 we compare results of using the $N_{f}=2 \mathrm{cA} 2.09 .48$ ensemble to the $N_{f}=2+1+1 \quad \mathrm{cB} 211.072 .64$ ensemble. The results are extracted from the plateau method with time separation $t_{s}=1.3 \mathrm{fm}$ for both isovector and isoscalar electric and magnetic form factors. We observe consistent results between the two ensembles. Therefore, to the accuracy of our data, no quenching effects due the strange and charm quarks can be detected. This corroborates our previous study where we found consistent results when comparing an $N_{f}=2$ and an $N_{f}=2+1+1$ ensemble at a pion mass of about $370 \mathrm{MeV}$ [21].

(iii) Isolation of ground state matrix element: An analysis of excited state contributions is carried out by 

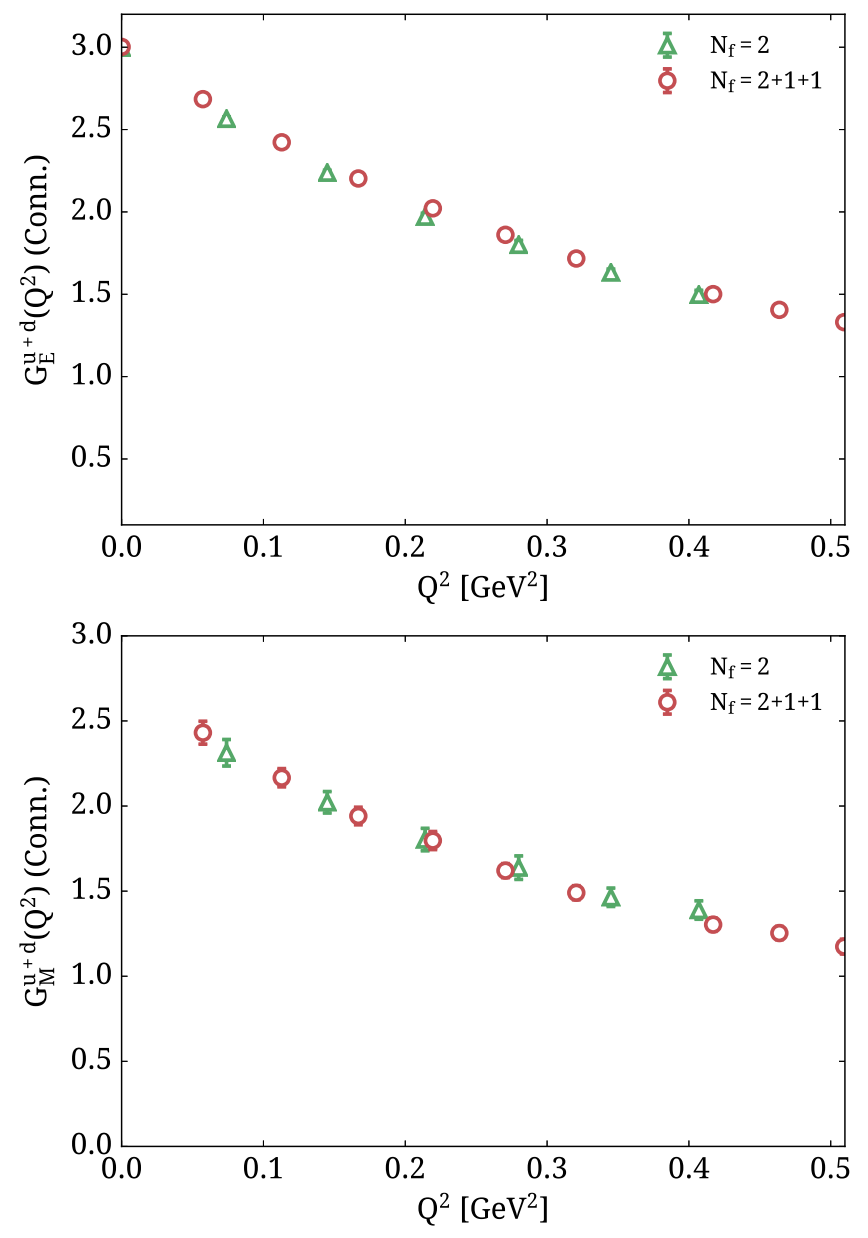

FIG. 12. Comparison of the connected contribution to the isoscalar electric (top panel) and magnetic (lower panel) form factors between the $N_{f}=2$ [7] and $N_{f}=2+1+1$ ensembles. The notation is the same as in Fig. 11.

performing the calculation using several time separations $t_{s}$. For the target $N_{f}=2+1+1$ ensemble we use five values of $t_{s}$ tabulated in Table II. We probe convergence to the ground state matrix element by demanding that the matrix element extracted using the plateau and two-state fits is consistent, as explained in detail in Sec. III C. The value of $t_{s} \sim 1.6 \mathrm{fm}$ is the largest utilized in this study and to our knowledge in any other study at the physical point. We increase the statistics as $t_{s}$ increases to keep the errors under control so that a meaningful analysis can be performed to isolate the ground state matrix element. For all of our results we observe agreement between the values extracted using the plateau and two-state fits. Despite this agreement, residual contamination can still lead to a systematic error within our current statistics. We give an estimate of such a systematic error by comparing the values obtained with the plateau and two-state fits.
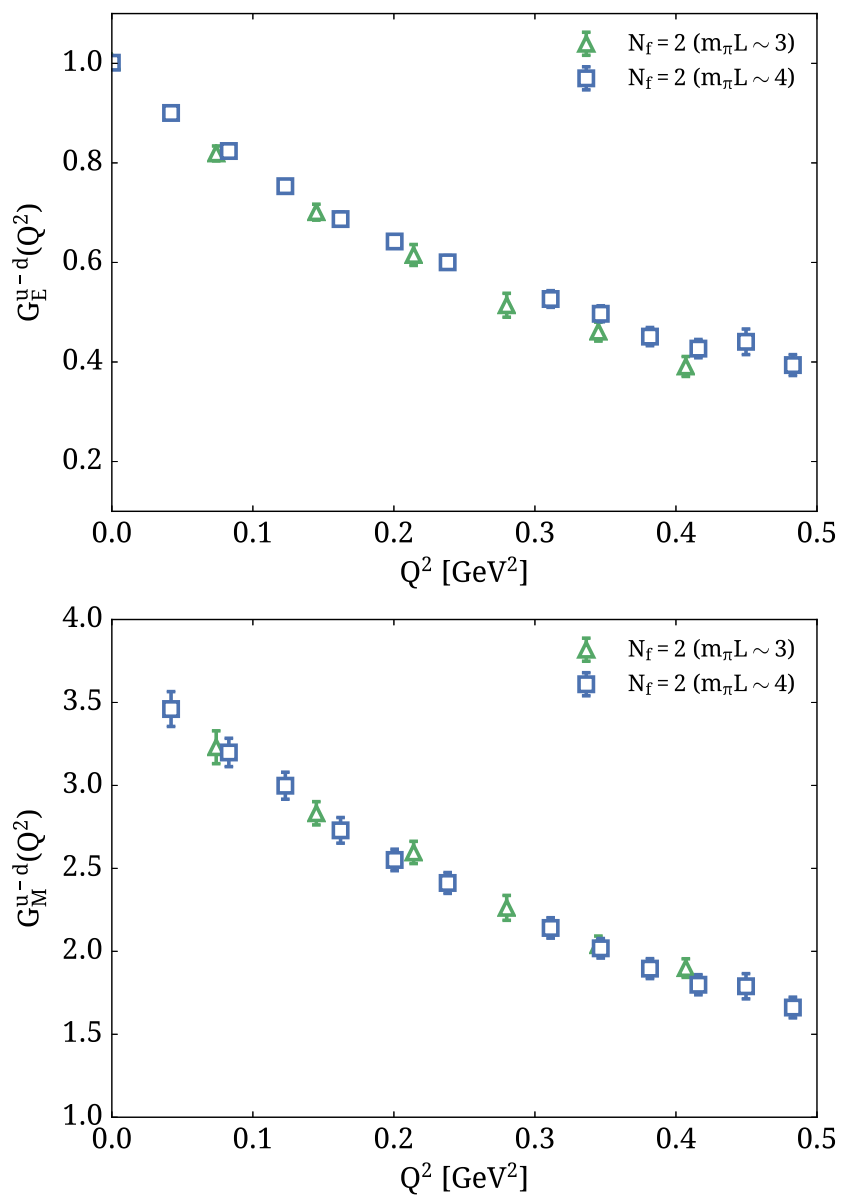

FIG. 13. The isovector electric (top panel) and magnetic (lower panel) form factors for two different physical spatial volumes. With green triangles we show the results for the cA2.09.48 with $m_{\pi} L \simeq 3$ [7] and with blue squares the cA2.09.64 with $m_{\pi} L \simeq 4$. Results are extracted using the plateau method for sink-source separation $t_{s} \simeq 1.5 \mathrm{fm}$ for the electric and $t_{s} \simeq 1.3 \mathrm{fm}$ for the magnetic form factors.

(iv) Finite volume effects: For the assessment of finite volume effects we compare the two $N_{f}=2$ physical point ensembles cA2.09.48 and cA2.09.64 that yield respectively $m_{\pi} L \simeq 3$ [7] and $m_{\pi} L \simeq 4$. The lattice spacing and pion mass are the same for these two ensembles. We also use the same time separation $t_{s}$ for each observable when comparing the two ensembles. The isovector electric and magnetic form factors extracted using the plateau method are shown in Fig. 13. The results fall on the same curve indicating no significant finite volume effects between the two volumes of $m_{\pi} L \simeq 3$ and $m_{\pi} L \simeq 4$. The same behavior is observed for the isoscalar form factors shown in Fig. 14. We would like to stress once more that our statement of detecting no volume effects can only be made within the current accuracy and some residual volume effects can still lead to a systematic effect. One complication as the volume 

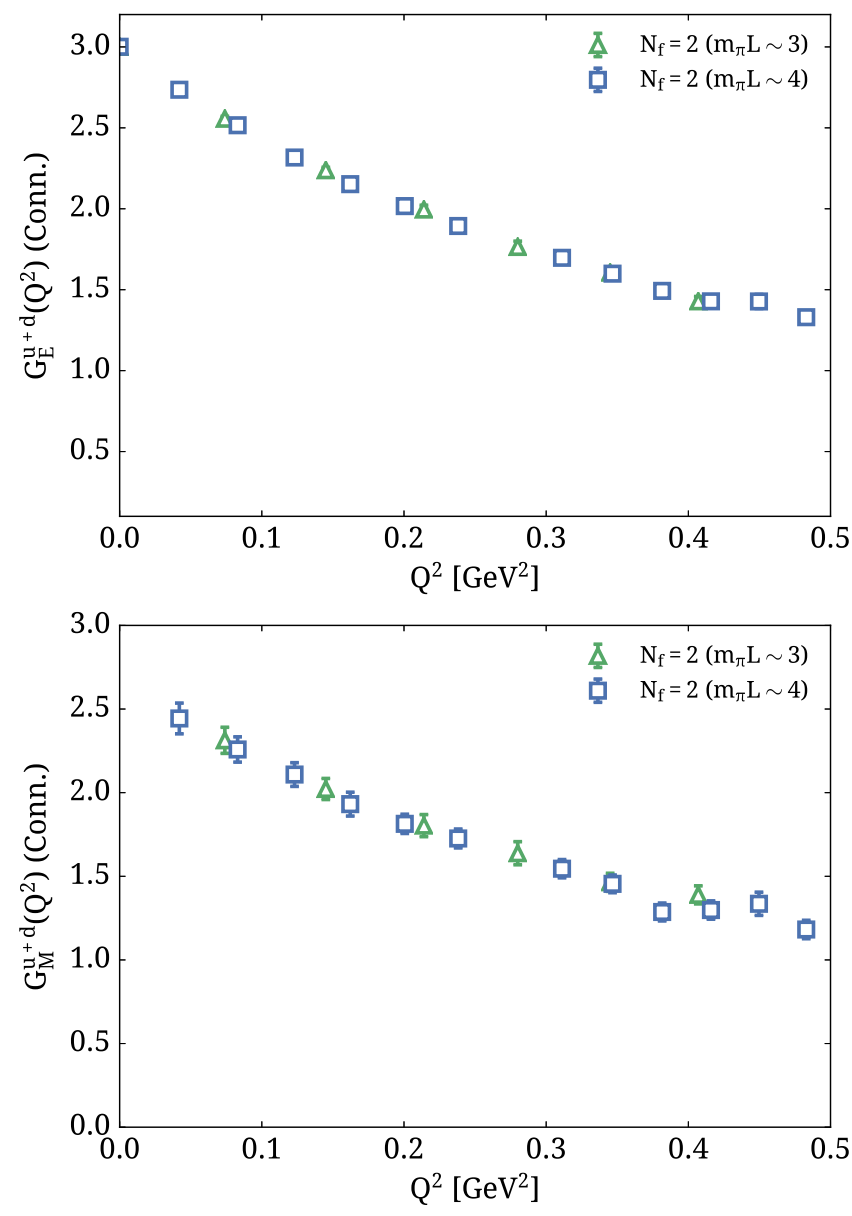

FIG. 14. The isoscalar electric (top panel) and magnetic (lower panel) form factors for two different physical spatial volumes. The notation is as in Fig. 13.

increases is the contamination due to higher excited states, since the number of multihadron states allowed increases [43]. Such multihadron states are not expected to affect results at larger pion masses but are expected to be more severe at the physical pion mass. Such effects can be modeled within chiral perturbation theory for the axial form factors [43]. For the electromagnetic form factors these effects are not known but an interplay between volume and excited state effects may account for the deviations observed between lattice QCD data and experimental values.

(v) Finite lattice spacing, a: Since in this work we are using the twisted mass formulation at maximal twist our results are automatically $\mathcal{O}(a)$ improved without any need to improve the current. This is different from clover fermions where the current must be improved in order to eliminate order $a$ contributions. Therefore, our results only have corrections of $\mathcal{O}\left(a^{2}\right)$. Continuum extrapolation cannot be performed given that we have analyzed only one $N_{f}=$ $2+1+1$ ensemble. The two $N_{f}=2$ ensembles analyzed have the same lattice spacing thus finite lattice spacing effects cannot be assessed. Previous studies done using ensembles with pion mass spanning about 460 to $260 \mathrm{MeV}$ and three values of the lattice spacings have indeed demonstrated that the $\mathcal{O}\left(a^{2}\right)$ correction is negligible [21]. We thus do not expect large systematic cutoff effects on our results. However, an analysis of cutoff effects will need to be carried out in the future when additional ensembles are available.

In summary, there maybe a slow convergence as a function of the volume in conjunction with residual excited state effects. This may explain the few $\sigma$ discrepancy observed between lattice QCD results and the experimental values. In particular, we note that the electric form factors have increasing excited state effects for larger values of $Q^{2}$, whereas for $G_{M}$ these effects are bigger at small $Q^{2}$. As we will see these are the ranges of momenta where we see discrepancies with the experimental values.

\section{VI. $Q^{2}$ DEPENDENCE OF THE ISOVECTOR AND ISOSCALAR FORM FACTORS}

In this section we discuss the $Q^{2}$ dependence of the form factors using standard parametrizations as described in the next section.

\section{A. Parametrizations of the $Q^{2}$ dependence}

Assuming vector meson pole dominance for $Q^{2}<0$, one expects that for small $Q^{2}>0$ the behavior will be dominated by the poles in the timelike region. One would then expect a dipole form given by [44]

$$
G\left(Q^{2}\right)=\frac{G(0)}{\left(1+\frac{Q^{2}}{M^{2}}\right)^{2}},
$$

where $M$ is the mass of the vector meson that parametrizes the $Q^{2}$ dependence. The value of the form factor at zero momentum transfer gives the electric charge in the case of the electric form factor and the magnetic moment in the case of the magnetic form factor. Combining Eqs. (19) and (5) one can relate $M$ to the mean square radius as

$$
\left\langle r^{2}\right\rangle=\frac{12}{M^{2}} .
$$

The neutron electric form factor and disconnected contributions to the electric form factors are zero for $Q^{2}=$ 0 and we treat them as special cases, fitting them using the Galster-like parametrization $[8,45]$ given by

$$
G\left(Q^{2}\right)=\frac{Q^{2} A}{4 m_{N}^{2}+Q^{2} B} \frac{1}{\left(1+\frac{Q^{2}}{0.71 \mathrm{GeV}^{2}}\right)^{2}},
$$

with $A$ and $B$ fit parameters. In this case the radius is given by 


$$
\left\langle r^{2}\right\rangle=-\frac{3 A}{2 m_{N}^{2}} .
$$

Another fit form, which has been applied recently to experimental data of both electromagnetic and axial form factors, is the model-independent z-expansion [9]. In this case, the form factor is expanded in a series given by

$$
G\left(Q^{2}\right)=\sum_{k=0}^{k_{\max }} a_{k} z^{k},
$$

where

$$
z=\frac{\sqrt{t_{\mathrm{cut}}+Q^{2}}-\sqrt{t_{\mathrm{cut}}}}{\sqrt{t_{\mathrm{cut}}+Q^{2}}+\sqrt{t_{\mathrm{cut}}}}
$$

and $t_{\text {cut }}$ is the timelike cut of the form factor. We take $t_{\text {cut }}=$ $4 m_{\pi}^{2}$ for the isovector combination $G^{u}-G^{d}$ and $t_{\text {cut }}=9 m_{\pi}^{2}$ for the isoscalar combination $G^{u}+G^{d}$ [9]. For convergence of the truncated series of Eq. (23), the coefficients $a_{k}$ should be bounded in size and convergence should be demonstrated by increasing $k_{\max }$. The interested reader is referred to Ref. [6] for details about our procedure. The mean square radius is given by

$$
\left\langle r^{2}\right\rangle=-\frac{3 a_{1}}{2 a_{0} t_{\mathrm{cut}}},
$$

while the value of the form factor at zero momentum transfer is $G(0)=a_{0}$.

\section{B. Fits to lattice $Q C D$ results}

We consider first the isovector form factors where only the connected diagram contributes. In Figs. 15 and 16 we

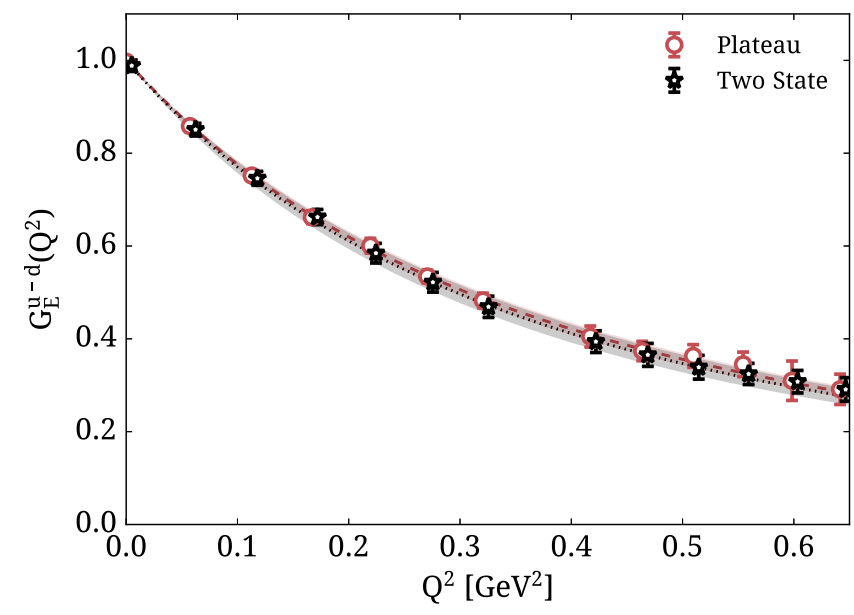

FIG. 15. $G_{E}^{u-d}\left(Q^{2}\right)$ from the plateau method for $t_{s} / a=20$ (circles) and two-state fits (stars). The dashed (dotted) curve and corresponding band is a dipole fit to the plateau (two-state) fit results, which overlap.

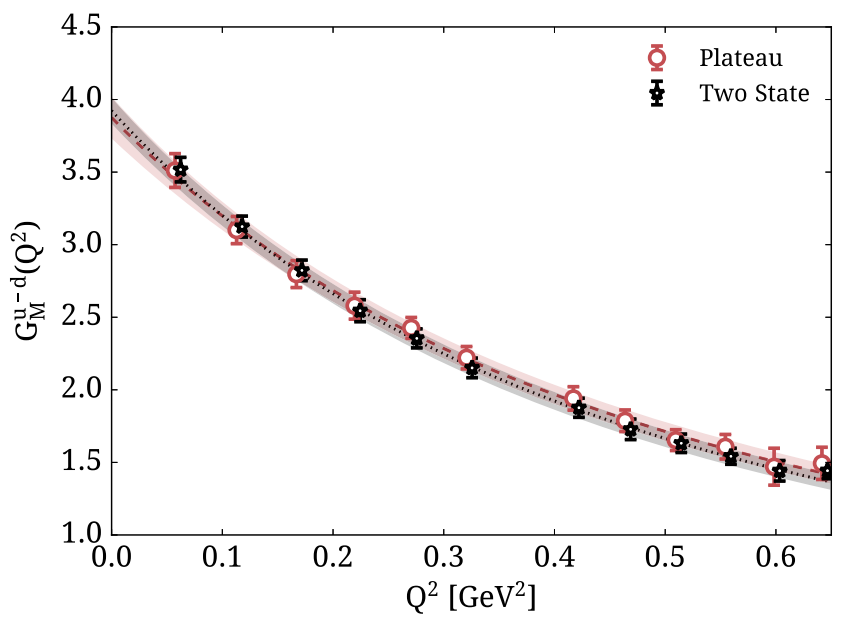

FIG. 16. $G_{M}^{u-d}\left(Q^{2}\right)$ from the plateau method for $t_{s} / a=20$ (circles) and two-state fits (stars). The rest of the notation is as in Fig. 15.

show fits using the dipole form, comparing between results from the plateau method at $t_{s} / a=20$ and from two-state fits for $G_{E}^{u-d}\left(Q^{2}\right)$ and $G_{M}^{u-d}\left(Q^{2}\right)$, respectively. As can be seen, fits using the plateau and two-state methods are fully consistent and do not show any significant systematic effect on the determination of the $Q^{2}$ dependence of the form factors, indicating that excited states are sufficiently

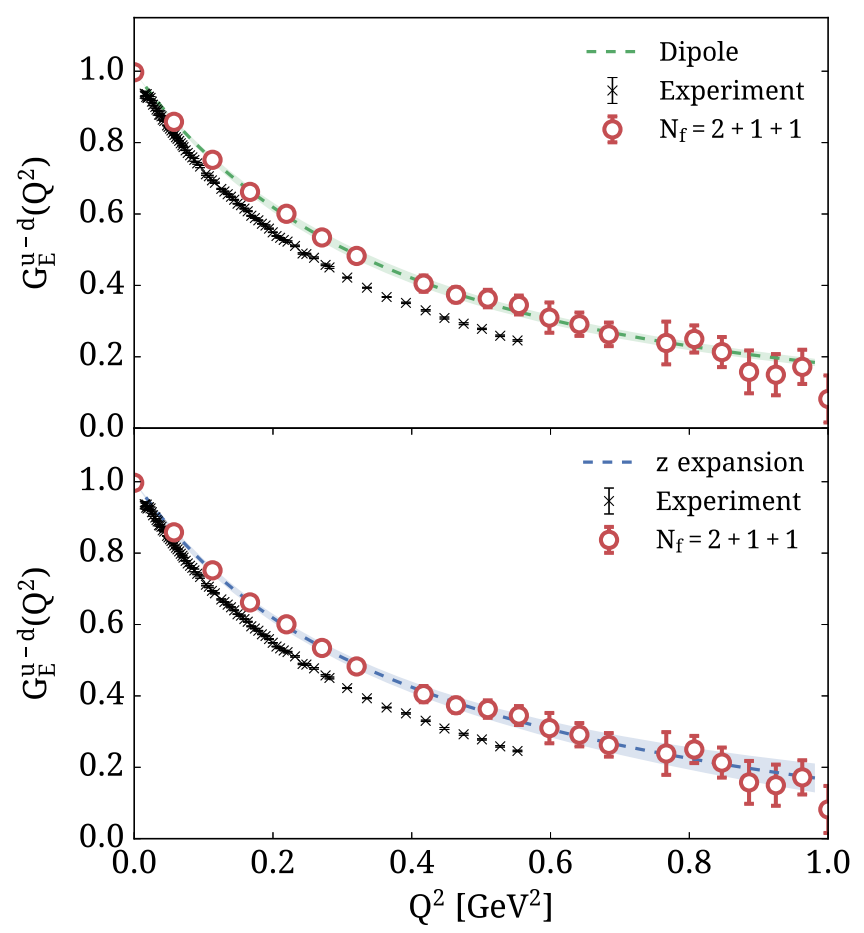

FIG. 17. The isovector electric form factor as a function of $Q^{2}$ (circles). We show fits to our results using a dipole form (top) and using the z-expansion (bottom) for $k_{\max }=4$. Black crosses are experimental results taken from the A1 Collaboration [1] for the proton and from Refs. [4,46-59] for the neutron. 


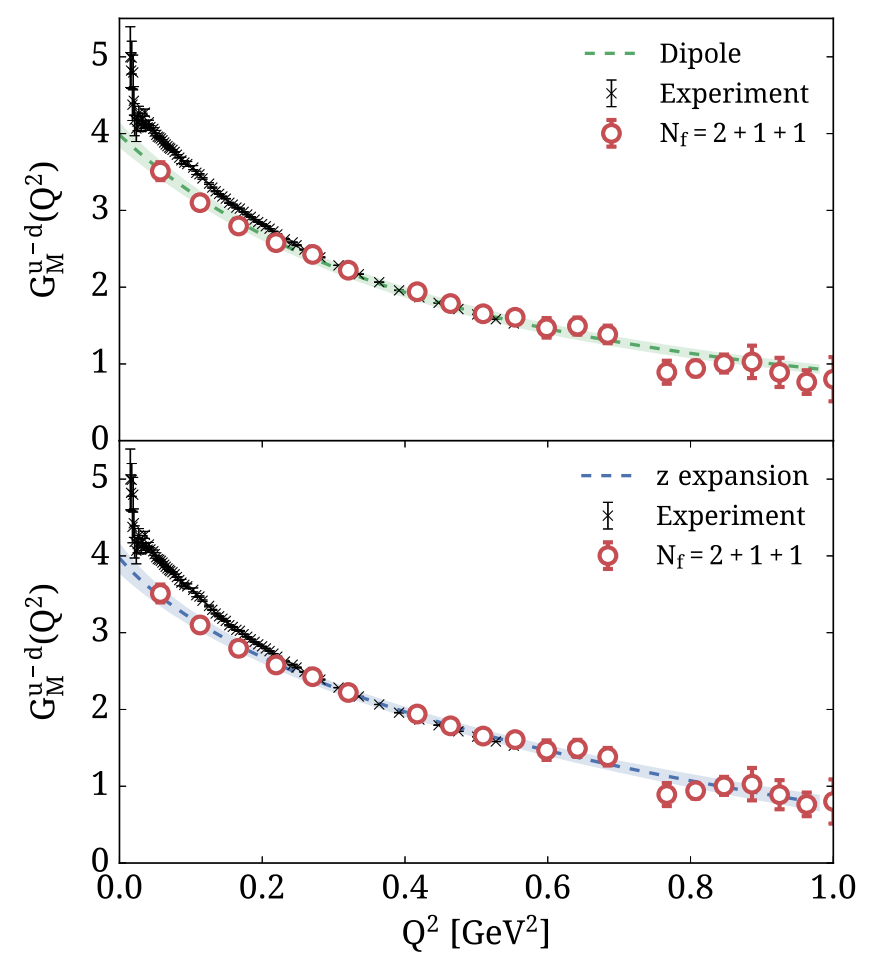

FIG. 18. The isovector magnetic form factor fitted using a dipole form (top) and using the z-expansion (bottom) with the notation of Fig. 17.

suppressed. Since results are in agreement, from now on we will use the plateau method at $t_{s} / a=20$ to extract final results on the form factors, rms radii, and magnetic moment. We will use the results extracted from the twostate fits to estimate the systematic error due to excited states.

In Figs. 17 and 18, we show fits to $G_{E}^{u-d}\left(Q^{2}\right)$ and $G_{M}^{u-d}\left(Q^{2}\right)$, respectively using the dipole form and the $\mathrm{z}$ expansion of Eq. (23) and compare to experiment. For the $\mathrm{z}$-expansion, we check convergence by increasing $k_{\max }$. The resulting magnetic moment and rms radii are shown in Fig. 19, where we observe convergence for $k_{\max }=4$. For $G_{E}^{u-d}\left(Q^{2}\right)$, we see from Fig. 17 that the slope of the lattice QCD data is less as compared to the experimental values. Therefore, although both dipole form and z-expansion describe very well our data shown in separate panels for clarity, they lie consistently above the experimental values. A study using a larger volume with a careful examination of excited state effects is planned to understand the origin of this remaining discrepancy. In extracting the rms radius, we see from Fig. 19 that results obtained from using the dipole fit and z-expansion are compatible, and yield

$$
\sqrt{\left\langle r_{E}^{2}\right\rangle^{u-d}}=0.796(19)(12)(12) \mathrm{fm},
$$

where the central value and the statistical error are taken from the dipole fit, the second error is systematic computed

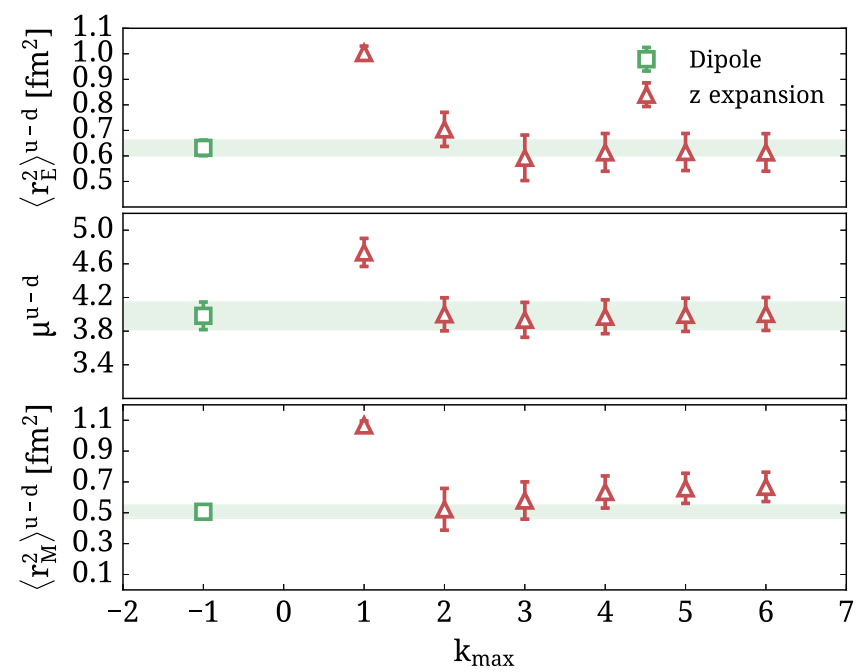

FIG. 19. Results for the isovector charge radius $\left\langle r_{E}^{2}\right\rangle^{u-d}$, magnetic moment $\mu^{u-d}$, and magnetic radius $\left\langle r_{M}^{2}\right\rangle^{u-d}$ from the plateau method using $t_{s} / a=20$ as extracted from a dipole fit (green square) and z-expansion (red triangles). The latter are shown as a function of $k_{\max }$. The green band is the statistical error on the value extracted from the dipole fit.

as the difference in the mean values between dipole and $\mathrm{z}$ expansion for $k_{\max }=4$, and the third error is the systematic error due to excited states obtained from the difference when fitting the form factor extracted from the plateau and from the two-state fit method. Subsequent quantities given in the paper will have statistical and systematic errors quoted using the same convention as in Eq. (26).

For $G_{M}^{u-d}$ shown in Fig. 18, we observe that our results are in agreement with the experimental values for $Q^{2}>0.2 \mathrm{GeV}^{2}$, whereas for small $Q^{2}$ they tend to be lower. A possible explanation for this discrepancy is that effects from the pion cloud expected to be prominent for small momenta [60] are suppressed in our calculation due to our finite volume. The fact that we have seen no volume effects when we increase the volume from $L m_{\pi} \simeq 3$ to $L m_{\pi} \simeq 4$ for our two $N_{f}=2$ ensembles may indicate that pion cloud suppression may not be detectable for these volume sizes requiring larger volumes to unfold. Indeed, preliminary results by PACS using a physical point ensemble with $L m_{\pi} \simeq 7.4$ [61] finds a higher value that may point to a finite volume effect. This would need further investigation to confirm.

The isovector magnetic moment and mean square magnetic radii are shown in Fig. 19. As can be seen, the mean values extracted for $\mu^{u-d}$ using the dipole and z-expansion are compatible, while for $\left\langle r_{M}^{2}\right\rangle^{u-d}$ the $\mathrm{z}$ expansion produces a slightly higher mean value, which, however, is consistent within errors. Quoting the values from the dipole fit, we find

$$
\mu^{u-d}=3.97(15)(2)(5)\left(\begin{array}{l}
1 \\
0
\end{array}\right)
$$




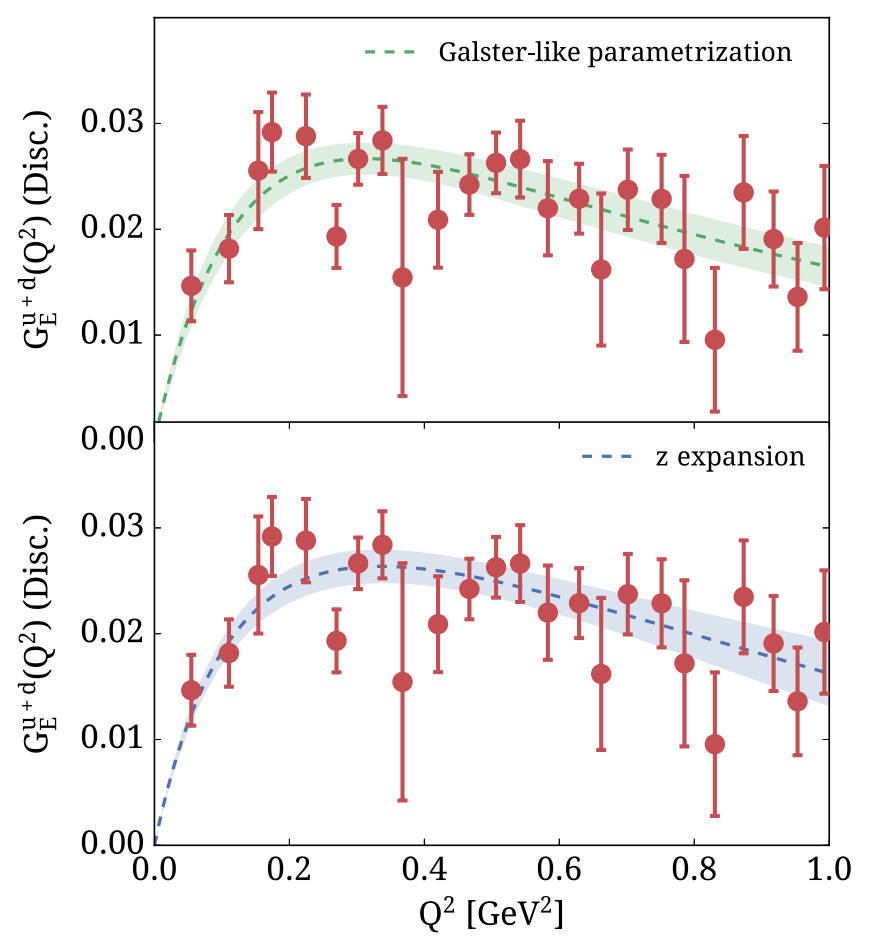

FIG. 20. Disconnected contributions to the isoscalar electric form factor (circles) as a function of $Q^{2}$. The fits using the Galster-like parametrization of Eq. (21) and the z-expansion for $k_{\max }=3$ are shown in the upper and lower panels, respectively.

$$
\sqrt{\left\langle r_{M}^{2}\right\rangle^{u-d}}=0.712(27)(87)(5)\left(\begin{array}{l}
1 \\
0
\end{array}\right) \mathrm{fm}
$$

Here we have included a fourth systematic error computed as the difference in the values of $\mu^{u-d}$ and $\left\langle r_{M}^{2}\right\rangle^{u-d}$ when fitting $G_{M}^{u-d}\left(Q^{2}\right)$ including and excluding the lowest $Q^{2}$ value from the fit. The error is asymmetric, since the expectation is that pion cloud effects will increase the value of the magnetic form factor. It is also small compared to the systematic error due to excited states. In what follows we will not include this fourth systematic error.

Before presenting fits to the total isoscalar form factors we discuss separately the $Q^{2}$ dependence of the disconnected contributions. In Fig. 20 we show the disconnected contribution to the isoscalar electric form factor $G_{E}^{u+d}$ accompanied by fits to the Galster-like parametrization and z-expansion. We note that in the case of the $\mathrm{z}$ expansion we take $a_{0}=0$, since $G_{E}^{u+d}(0)=0$ for the disconnected contribution. Both parametrizations describe well our results with the z-expansion yielding a larger error for the larger $Q^{2}$ values.

The disconnected contribution to $G_{M}^{u+d}\left(Q^{2}\right)$ is shown in Fig. 21. We find that both dipole and z-expansion are in good agreement. In particular, they yield compatible values at zero momentum transfer. Like in the case of the disconnected contribution to $G_{E}^{u+d}\left(Q^{2}\right)$, for large $Q^{2}$ the dipole fit has a smaller error band as compared to

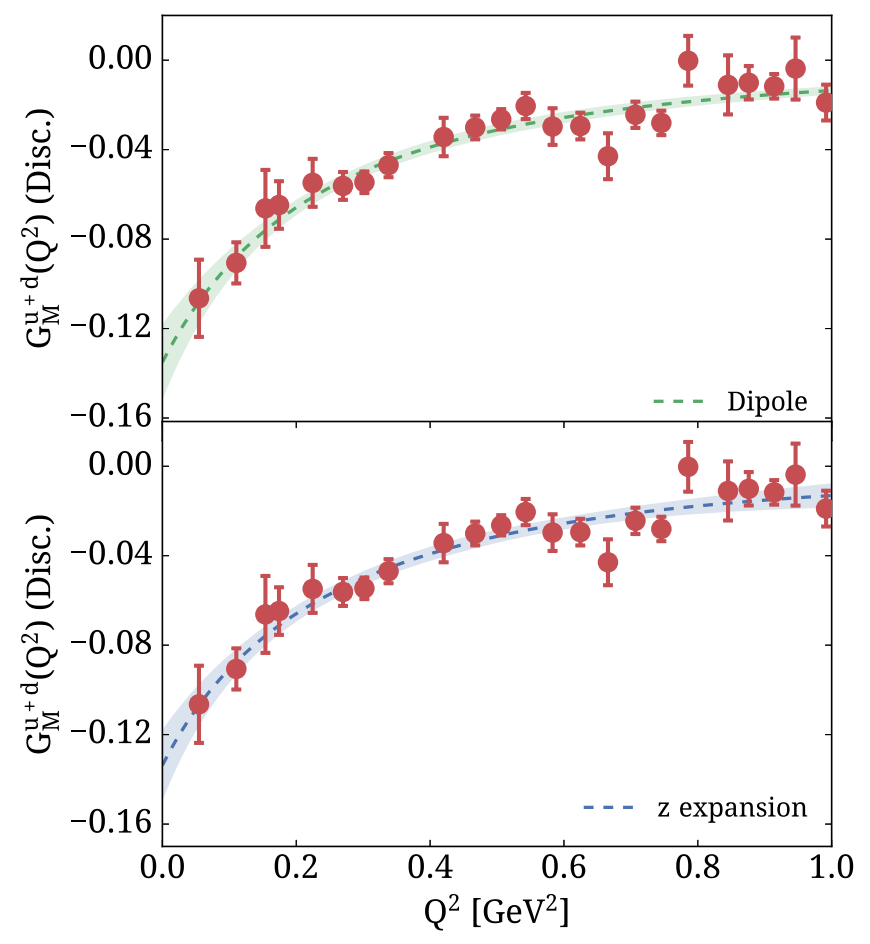

FIG. 21. Disconnected contributions to the isoscalar magnetic form factor. The notation is the same as in Fig. 20.

the z-expansion. The values extracted from fitting the disconnected contributions alone are

$$
\begin{gathered}
\left\langle r_{E}^{2}\right\rangle^{u+d}(\text { Disc })=-0.071(6)(4)(6) \mathrm{fm}^{2}, \\
\mu^{u+d}(\text { Disc })=-0.134(17)(1)(10), \\
\left\langle r_{M}^{2}\right\rangle^{u+d}(\text { Disc })=-0.136(30)(2)(12) \mathrm{fm}^{2},
\end{gathered}
$$

where we have not normalized with the value of the form factor at zero momentum transfer; i.e., the radii are extracted from $\left\langle r^{2}\right\rangle=-\left.6 \frac{\partial G\left(Q^{2}\right)}{\partial Q^{2}}\right|_{Q^{2}=0}$ rather than from Eq. (5).

In Fig. 22 we show the isoscalar form factors when including and excluding disconnected contributions. Although the effect is small for both $G_{E}^{u+d}\left(Q^{2}\right)$ and $G_{M}^{u+d}\left(Q^{2}\right)$ there is a systematic shift affecting the parameters of the fits. This comparison shows that disconnected contributions although small are important to include and that their omission would result in an uncontrolled systematic error comparable to the statistical uncertainty. Such systematics need to be under control for precision results required for distinguishing e.g., the two experimental determinations of the charge radius of the proton.

In Figs. 23 and 24 we show the fits of the total isoscalar electric and magnetic form factors using the dipole form and z-expansion. Both fits describe well the data with the dipole fit being more precise at larger $Q^{2}$, a behavior also observed for the isovector form factors. For intermediate 


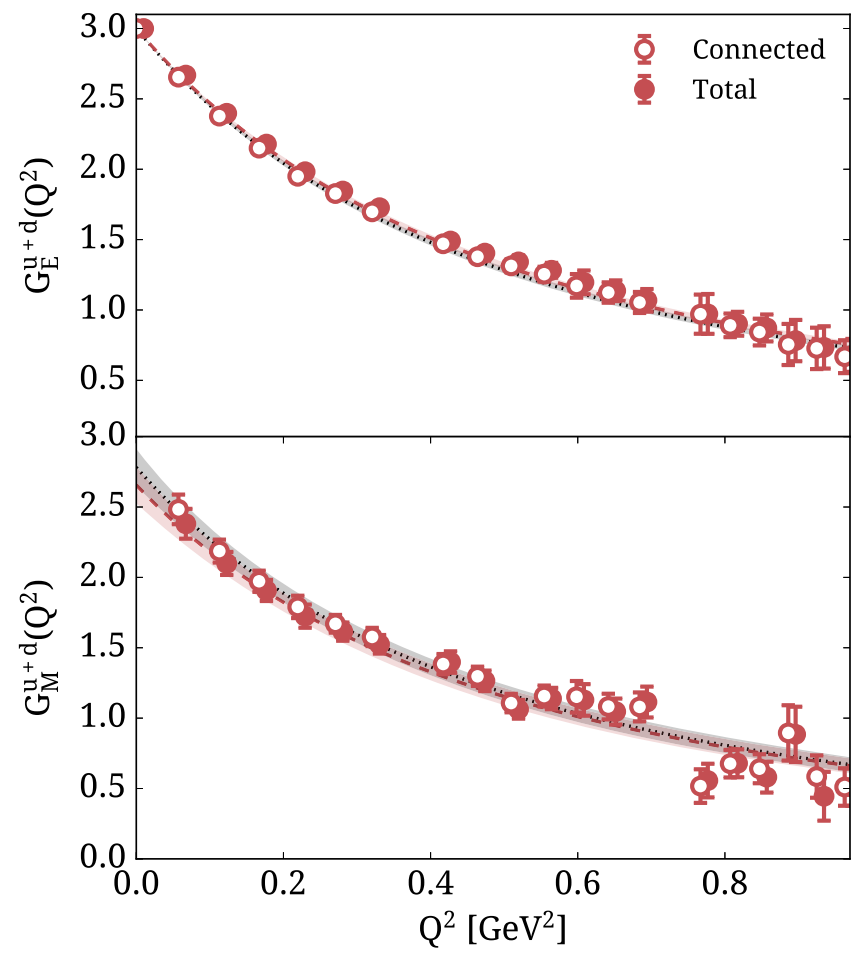

FIG. 22. Comparison of the connected (open circles) and total (filled circles) contributions to the isoscalar electric (top) and magnetic (bottom) form factors. Dipole fits to the connected and total contributions are shown with the dotted and dashed curves respectively.

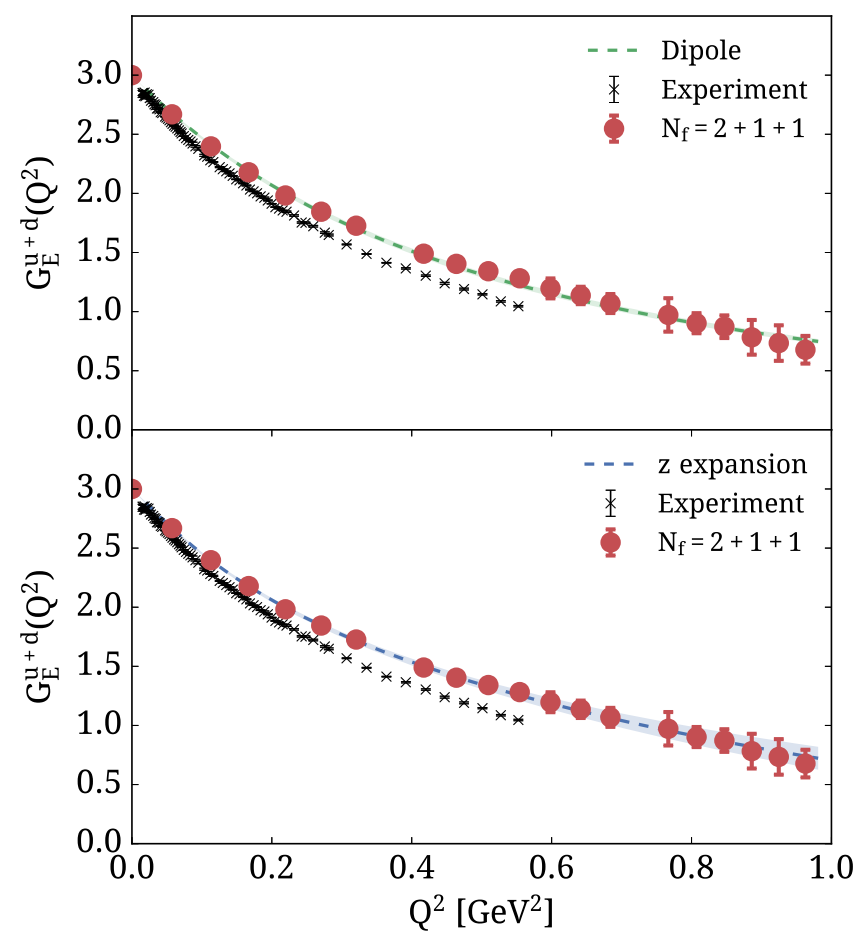

FIG. 23. Isoscalar electric form factor (circles) as a function of $Q^{2}$. We combine the connected contribution from the plateau for $t_{s} / a=20$ with the disconnected contribution for $t_{s} / a=14$. The remaining notation is as in Fig. 17.

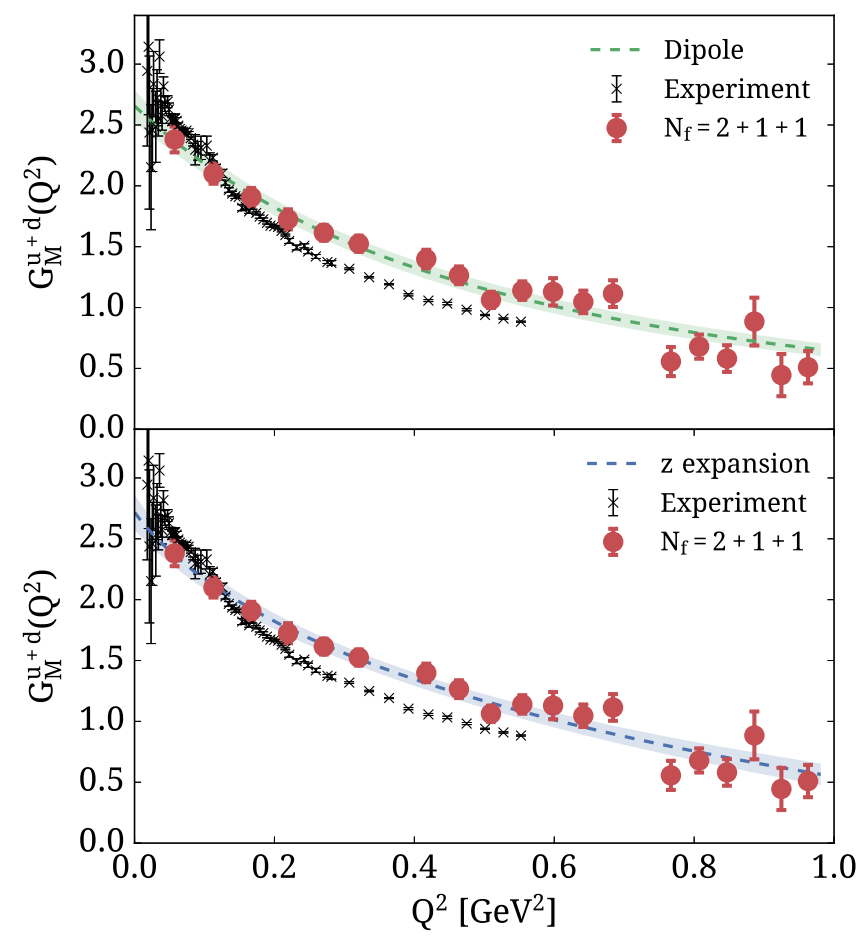

FIG. 24. Isoscalar magnetic form factor. The notation is as in Fig. 23.

$Q^{2}$ values our results are systematically higher compared to experiment, which is then reflected in the fit bands. Since for low $Q^{2}$ there is agreement, the extracted value for the isoscalar magnetic moment agrees with the experimental value. On the other hand, the slope of our lattice data is not as steep as in the experimental results, which leads to a smaller value for the corresponding radii.

In the top panel of Fig. 25 we show the isoscalar electric square radius. As can be seen, the z-expansion fit yields

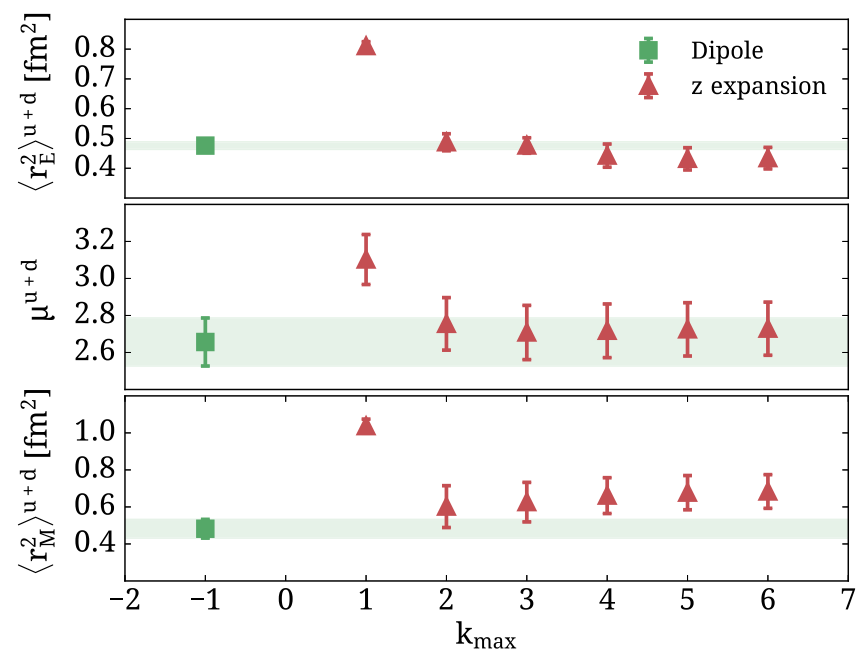

FIG. 25. Results for the isoscalar charge square radius, magnetic moment, and magnetic square radius. The notation is as in Fig. 19. 
values that are within errors for $k_{\max }>1$ but with twice larger errors than the dipole. In Fig. 25 we also show results for the magnetic moment and the magnetic radius where convergence of the z-expansion is observed already for $k_{\max }=2$. In general, there is agreement between the results extracted from the dipole and z-expansion. In what follows we will quote the values determined from the dipole fits and quote as a systematic error the difference between the mean values of the dipole and the z-expansion fits. We find

$$
\begin{gathered}
\sqrt{\left\langle r_{E}^{2}\right\rangle^{u+d}}=0.691(9)(7)(14) \mathrm{fm}, \\
\mu^{u+d}=2.66(13)(9)(9), \quad \text { and } \\
\sqrt{\left\langle r_{M}^{2}\right\rangle^{u+d}}=0.695(33)(80)(13) \mathrm{fm} .
\end{gathered}
$$

Note that from our definition of the isoscalar combination, the proton plus neutron magnetic moment is obtained by $\mu^{p}+\mu^{n}=\mu^{u+d} / 3$.

\section{COMPARISON WITH OTHER STUDIES}

Before we discuss our final results for the proton and neutron form factors we compare with the results from other groups using different lattice QCD ensembles and discretization schemes. These mainly exist for the isovector electromagnetic form factors allowing us to qualitatively assess lattice artifacts. This is useful since most groups use a single ensemble and thus infinite volume and continuum extrapolations are lacking. We summarize the lattice QCD discretized actions used by different groups for the computation of the electromagnetic form factors, restricting ourselves only to published works and results that were obtained using simulations with pion mass less than $170 \mathrm{MeV}$ :

(i) LHPC analyzed one ensemble of $N_{f}=2+1$ with two levels of HEX-smeared clover fermions with $m_{\pi}=149 \mathrm{MeV}$, lattice spacing $a=0.116 \mathrm{fm}$, and $L m_{\pi}=4.21$ at three sink-source time separations from 0.93 to $1.39 \mathrm{fm}$ [13]. They give as their final results the ones extracted using the summation method, which leads to larger statistical errors. Additionally, they analyzed an $N_{f}=2+1$ ensemble with two levels of HEX-smeared clover fermions with $m_{\pi}=135 \mathrm{MeV}$, lattice spacing $a=0.093 \mathrm{fm}$, and $L m_{\pi}=4$ [14]. They analyzed three lattice separations from 0.93 to $1.5 \mathrm{fm}$ and they have extracted results using the summation method. A momentum derivative method has been used to extract the magnetic moment and the electric radius directly from the correlation functions avoiding a fitting procedure.

(ii) The PACS Collaboration analyzed an ensemble of $N_{f}=2+1$ stout-smeared clover fermions with $m_{\pi}=146 \mathrm{MeV}, a \simeq 0.085 \mathrm{fm}$, and a spatial extent of $8.1 \mathrm{fm}$ or $L m_{\pi} \simeq 6$ allowing access to relatively small momenta $[15,61]$. PACS has computed threepoint functions for one sink-source time separation of $1.27 \mathrm{fm}$ and they used the plateau method to identify the ground state matrix element.

(iii) The $\chi \mathrm{QCD}$ Collaboration [62] computed only the disconnected contributions to the nucleon electromagnetic form factors using a hybrid action of overlap valence quarks and $N_{f}=2+1$ domain wall sea quarks produced by RBC/UKQCD. Their analysis includes an ensemble with pion mass $m_{\pi}=139 \mathrm{MeV}, \quad a=0.1141(2) \mathrm{fm}$, and $L m_{\pi}=$ 3.86. They computed nucleon two-point functions stochastically using $Z_{3}$-noise grid sources and disconnected quark loops with $Z_{4}$-noise grids applying even-odd and time dilution as well as low-mode average.

(iv) Our results obtained using the three ensembles of Table I simulated by the Extended Twisted Mass Collaboration (ETMC). These include the two analyses of this work, namely the $N_{f}=2+1+1$ ensemble with $m_{\pi}=139 \mathrm{MeV}, a=0.0801(4)(3) \mathrm{fm}$, and $L m_{\pi} \simeq 3.6$ and the $N_{f}=2 \mathrm{cA} 2.09 .64$ ensemble with $m_{\pi}=130(1) \mathrm{MeV}, a=0.0938(3)(1) \mathrm{fm}$, and $L m_{\pi} \simeq 4$ as well as our results from Refs. [6,7], which were obtained using the $N_{f}=2 \mathrm{cA} 2.09 .48$ ensemble with $L m_{\pi} \simeq 3$ and the same pion mass and lattice spacing.

In Fig. 26 we show a comparison of lattice QCD results for $G_{E}^{u-d}\left(Q^{2}\right)$ up to $Q^{2}=0.5 \mathrm{GeV}^{2}$ from the analyses mentioned above. As can be seen, the ETMC and PACS results are in good agreement but systematically higher than the experimental values. LHPC results were obtained using the summation method and in general have larger statistical errors making them compatible with both our results and the experimental values.

In Fig. 26, we also show the corresponding results for $G_{M}^{u-d}\left(Q^{2}\right)$. The ETMC results of this work are the most precise and in good agreement with those obtained from other studies. We note the very good agreement of lattice QCD results and experiment for $Q^{2}>0.2 \mathrm{GeV}^{2}$. As pointed out, the underestimation of lattice $\mathrm{QCD}$ results compared to experimental values at smaller $Q^{2}$ may indicate that a larger spatial volume is required to develop fully the pion contributions. Although our study using two ensembles of $N_{f}=2$ showed no detectable volume effects when we increase the spatial extent from 4.5 to $6 \mathrm{fm}$ (or equivalently from $L m_{\pi} \sim 3$ to $L m_{\pi} \sim 4$ ) the volume dependence could be weak and require a larger volume to manifest itself. The new PACS results may indicate such a trend [61]. A conclusion that we can, however, draw from these lattice QCD studies is that there is agreement among them for both the electric and magnetic form factors. Given the different discretization schemes employed, this 


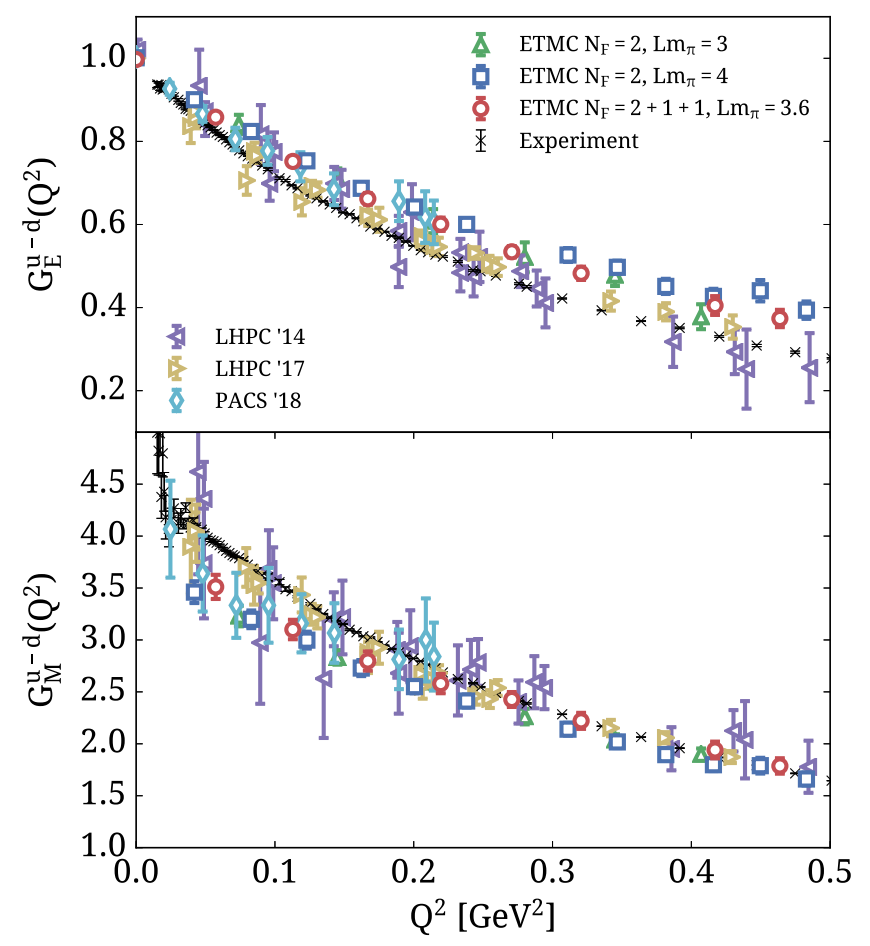

FIG. 26. Comparison of results for $G_{E}^{u-d}\left(Q^{2}\right)$ (upper panel) and $G_{M}^{u-d}\left(Q^{2}\right)$ (lower panel) from the $N_{f}=2+1+1$ twisted mass ensemble of this work (red circles), the $N_{f}=2$ twisted mass ensemble with $m_{\pi} L \simeq 4$ of this work (blue squares), the $N_{f}=2$ twisted mass ensemble with $m_{\pi} L \simeq 3$ from Ref. [7] (green triangles), LHPC using $N_{f}=2+1$ stout-smeared clover fermions from Ref. [13] (left purple triangles) and Ref. [14] (right yellow triangles), and from PACS using $N_{f}=2+1$ stoutsmeared clover fermions from Ref. [15] (cyan rhombuses).

agreement indicates that cutoff effects are smaller than the statistical errors.

In Fig. 27 we show a comparison of the disconnected contributions to $G_{E}^{u+d}$ and $G_{M}^{u+d}$ using results obtained from our $N_{f}=2+1+1$ and $N_{f}=2$ twisted mass ensembles and from the hybrid action as analyzed by the $\chi \mathrm{QCD}$ Collaboration [62]. We would like to stress the accuracy of the results of the current work using the $N_{f}=2+1+1$ twisted mass ensemble. In our previous evaluation of the disconnected contributions for the $N_{f}=2$ twisted mass ensemble we used 2120 configurations with 100 source positions for the computation of the two-point functions and 2250 stochastic vectors for the disconnected loops [6]. This is approximately the same number of inversions (and thus cost) as for the $N_{f}=2+1+1$ ensemble (see Table III), which demonstrates the effectiveness of the hierarchical probing method employed in the current analysis of the $N_{f}=$ $2+1+1$ ensemble.

The proton and neutron form factors can be extracted from the isovector and isoscalar form factors discussed in Sec. VI, using the linear combinations

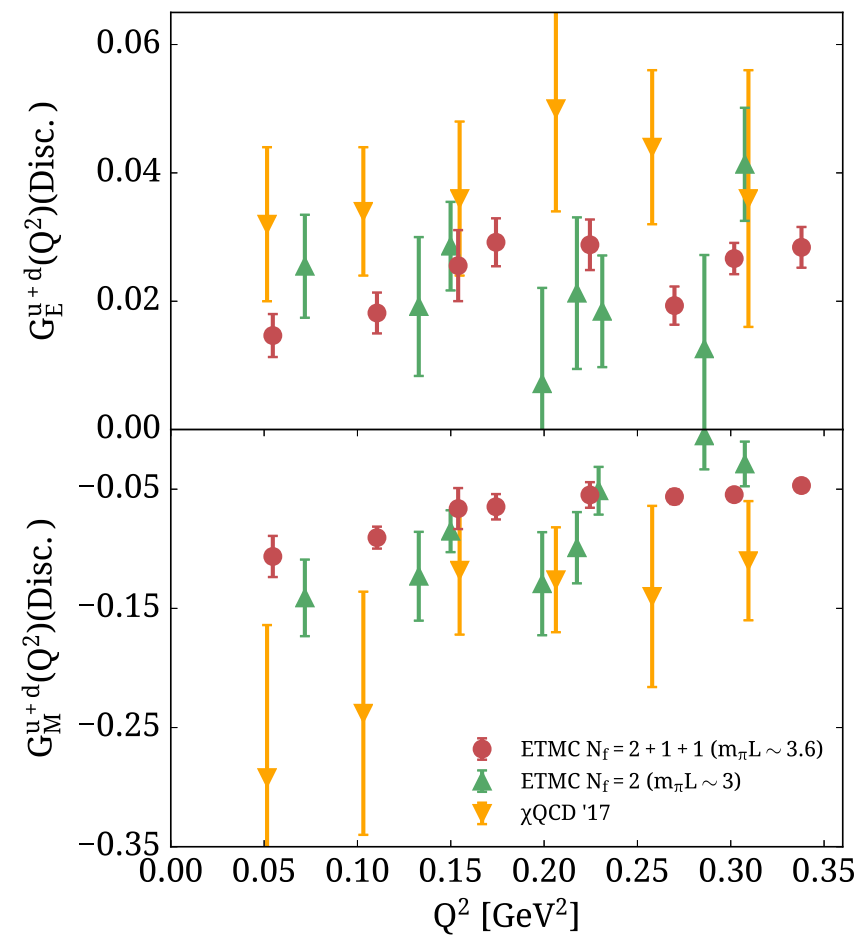

FIG. 27. Comparison of the disconnected contributions to $G_{E}^{u+d}$ (top) and $G_{M}^{u+d}$ (bottom) from the twisted mass ensemble using $N_{f}=2+1+1$ from this work (circles) compared to the twisted mass ensemble of $N_{f}=2$ results of Ref. [6] (up triangles) and the results from the $\chi \mathrm{QCD}$ Collaboration (down triangles) from Ref. [62].

$$
\begin{aligned}
& G^{p}\left(Q^{2}\right)=\frac{1}{2}\left[\frac{G^{u+d}\left(Q^{2}\right)}{3}+G^{u-d}\left(Q^{2}\right)\right], \\
& G^{n}\left(Q^{2}\right)=\frac{1}{2}\left[\frac{G^{u+d}\left(Q^{2}\right)}{3}-G^{u-d}\left(Q^{2}\right)\right] .
\end{aligned}
$$

In Fig. 28, we show lattice QCD results for the proton electromagnetic form factors. To extract these, one needs both the isovector and isoscalar combinations. The latter includes disconnected contributions, which have only been computed by ETMC for ensembles with physical pion masses. We still provide a comparison with the lattice results by LHPC which however do not include these disconnected contributions. We use filled symbols to indicate lattice QCD results that include disconnected contributions. For both the proton electric and magnetic form factors the LHPC results are in agreement with ours, with the LHPC results exhibiting larger errors due to the usage of the summation method. The accurate ETMC results are higher than the experimental values for $G_{E}^{p}\left(Q^{2}\right)$, while for $G_{M}^{p}\left(Q^{2}\right)$ they are in agreement except for the two lowest $Q^{2}$ values. Unfortunately, LHPC results carry large errors and in general are compatible both with our values and the experimental ones prohibiting any 


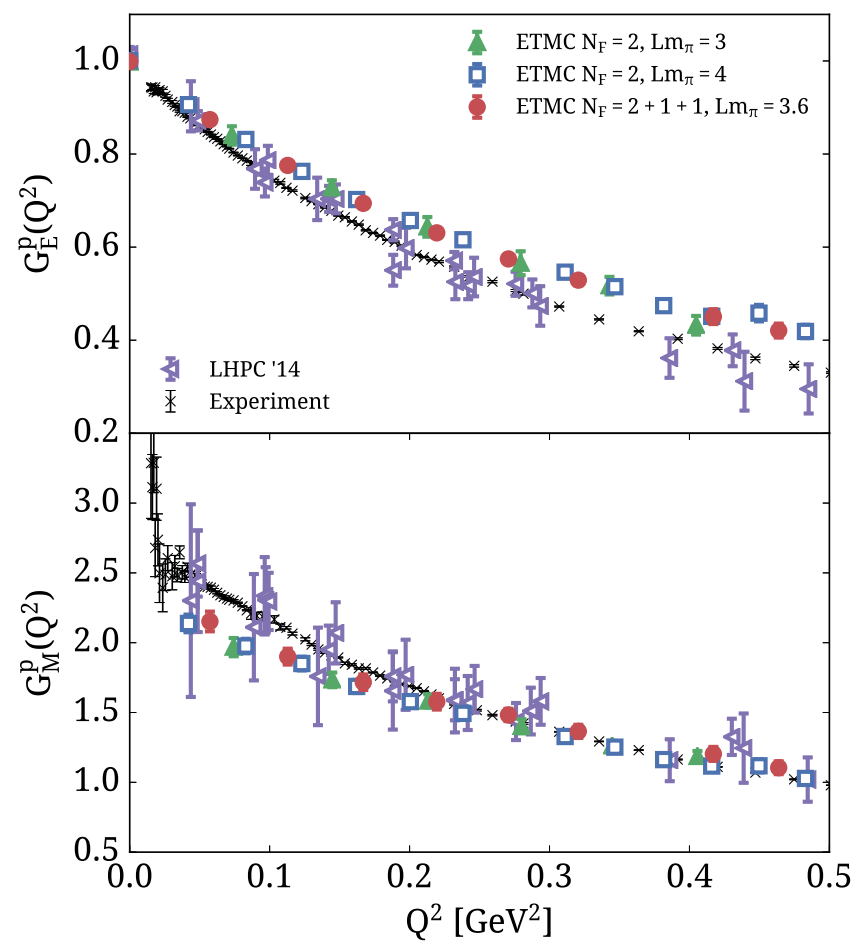

FIG. 28. Comparison of results for $G_{E}^{p}\left(Q^{2}\right)$ (upper panel) and $G_{M}^{p}\left(Q^{2}\right)$ (lower panel) from ETMC and LHPC following the notation of Fig. 26. Filled symbols are used for results that include disconnected contributions and open symbols for results without disconnected contributions. Black crosses are experimental results from the A1 Collaboration [1].

definite conclusions as to the nature of the discrepancy with the experimental values. As discussed volume and residual excited state effects may lead to a slow convergence of the lattice data that can account for the discrepancies with the experimental values.

Results for the neutron electromagnetic form factors are only provided by the ETMC for pion masses below $170 \mathrm{MeV}$. They are compared to the experimental values in Fig. 29. We observe that results for the electric form factor extracted from the $\mathrm{cB} 211.072 .64$ ensemble that includes disconnected contributions are in agreement with the experimental values. This is also true for the cA2.09.48 ensemble that includes disconnected contributions although they carry larger errors. For the cA2.09.64 ensemble, where disconnected contributions have not been included, the electric neutron form factor is underestimated. This clearly indicates the significance of including disconnected contributions, especially for this quantity, an observation consistent with the conclusion reached also in Ref. [62]. For the magnetic form factor, the results using the cB211.072.64 twisted mass ensemble with disconnected contributions are closer to experiment as to compared to the $N_{f}=2$ ensembles, but there is still a discrepancy with the experiment for small $Q^{2}$ values that needs to be further investigated.

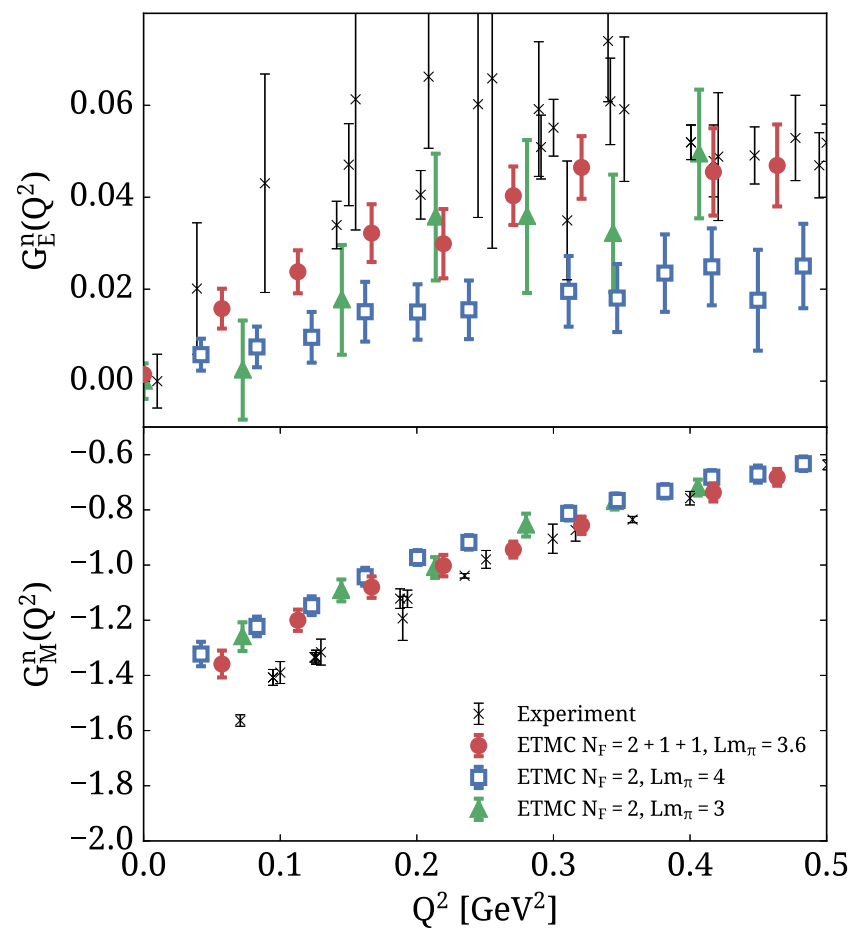

FIG. 29. Comparison of results for $G_{E}^{n}\left(Q^{2}\right)$ and $G_{M}^{n}\left(Q^{2}\right)$ using the $N_{f}=2+1+1$ results of this work (red circles), using the $N_{f}=2$ results with $m_{\pi} L \simeq 4$ of this work (blue squares), and using the $N_{f}=2$ ensemble with $m_{\pi} L \simeq 3$ from Ref. [6] (green triangles). Filled symbols are used for results that include disconnected contributions and open symbols for results without disconnected contributions. Crosses are experimental results taken from Refs. [4,46-59] for the electric form factor and from Refs. [63-68] for the magnetic form factor.

In Fig. 30, we compare the lattice QCD values of the isovector rms radii $\sqrt{\left\langle r_{E}^{2}\right\rangle^{u-d}}$ and $\sqrt{\left\langle r_{M}^{2}\right\rangle^{u-d}}$ finding agreement among them. As expected by the less steep falloff of the electric isovector form factor, lattice QCD results are systematically lower than the experimental values. We note that the ETMC results have errors that are already the same as the difference between the two experimental determinations showing that the statistical accuracy required can be achieved. A high-statistics dedicated study to better assess the remaining systematics can thus yield valuable insights on the rms charge radius from a first principles calculation. In the case of $\left\langle r_{M}^{2}\right\rangle^{u-d}$ the errors are larger and lattice QCD results are both in good agreement among them and compatible with the Particle Data Group (PDG) value [70].

In Fig. 31 we show the corresponding quantities for the proton. Only the ETMC results include disconnected contributions, which, although small, have a systematic effect. We observe a similar behavior as for the isovector case, namely smaller values for the electric and magnetic rms radii. LHPC results extracted using the summation method have larger errors and are thus compatible with 


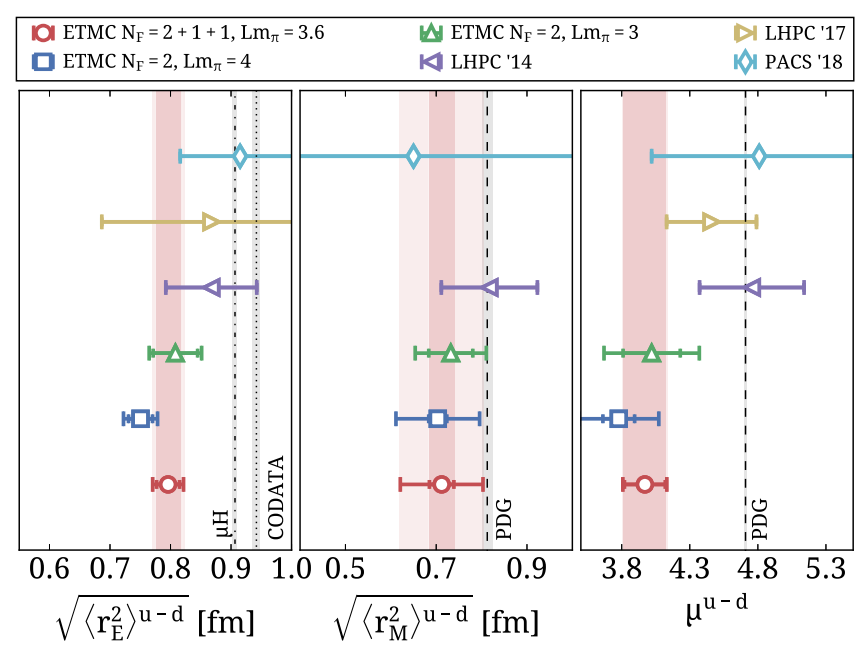

FIG. 30. Isovector $\sqrt{\left\langle r_{E}^{2}\right\rangle^{u-d}}, \sqrt{\left\langle r_{M}^{2}\right\rangle^{u-d}}$, and $\mu^{u-d}$ with lattice QCD results following the notation of Fig. 26. The experimental result extracted from muonic hydrogen [3] is shown by the vertical dashed-dotted line and from CODATA [69] by the dotted vertical line. The PDG value [70] is shown with the dashed vertical line. The red vertical inner band denotes the statistical error extracted using the $N_{f}=2+1+1$ twisted mass ensemble of this work and the outer lighter band is the total error adding statistical and systematic errors in quadrature.

both the muonic and electron scattering determinations of the rms radii. For the neutron radii we have only results from ETMC and LHPC. They are displayed in Fig. 32. ETMC results on the electric rms radius are determined at high accuracy and include all contributions. Although they are still smaller in magnitude than the experimental values, the discrepancy is within 1 standard deviation. We note that including disconnected contributions brings better agreement in particular in the case of $\left\langle r_{E}^{2}\right\rangle^{n}$.

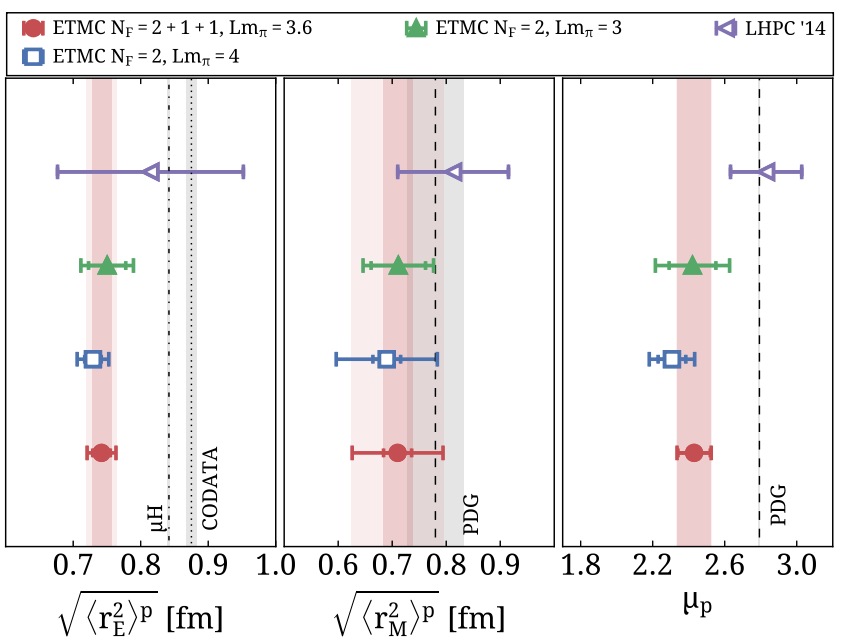

FIG. 31. Results for $\sqrt{\left\langle r_{E}^{2}\right\rangle^{p}}$ and $\sqrt{\left\langle r_{M}^{2}\right\rangle^{p}}$ using the same notation as in Fig. 30. Filled symbols denote results that include all contributions whereas open symbols are those where disconnected contributions are neglected. The rest of the notation follows that of Fig. 30.

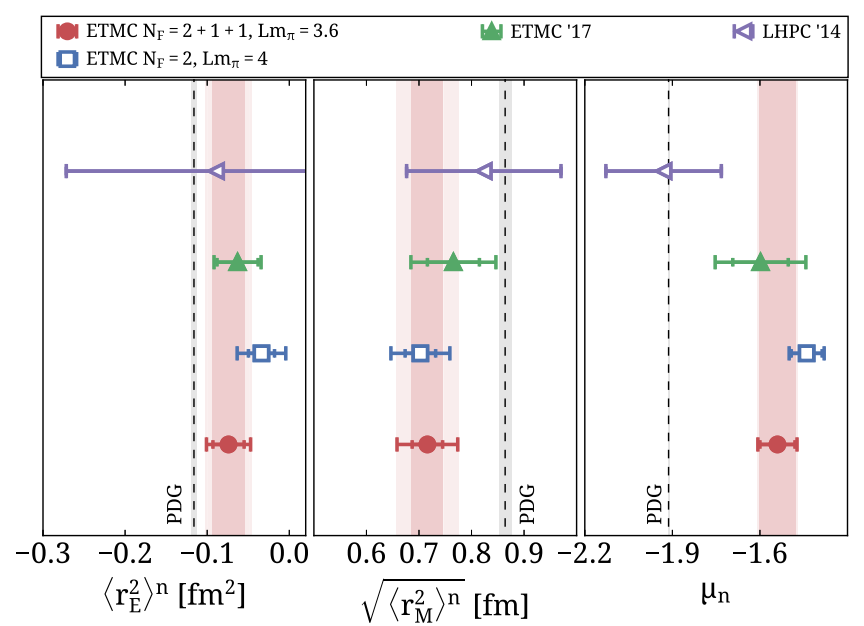

FIG. 32. Lattice QCD results for $\left\langle r_{E}^{2}\right\rangle^{n}, \sqrt{\left\langle r_{M}^{2}\right\rangle^{n}}$, and $\mu_{n}$. The notation is as in Fig. 31.

\section{PROTON AND NEUTRON ELECTROMAGNETIC FORM FACTORS}

Having compared with other groups and with the $N_{f}=2$ results from ETMC, we collect here our final results on the proton and neutron form factors using the $N_{f}=2+1+1$ ensemble, which has the most accurate results at the physical point. In Fig. 33 we show our results for the proton electric and magnetic form factors compared to

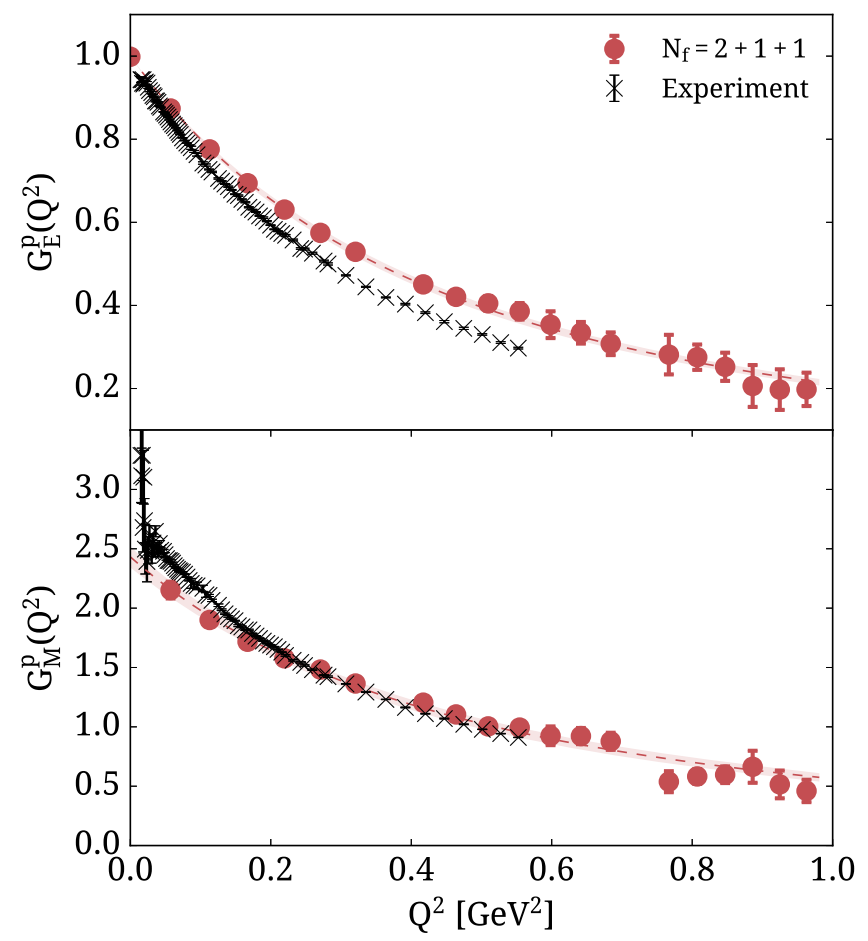

FIG. 33. Proton electric (upper panel) and magnetic (lower panel) form factors as a function of $Q^{2}$. Filled circles show the lattice QCD results of this work and black crosses are experimental results from the A1 Collaboration [1]. The band is the fit to our results using Eq. (19). 
experimental data. As expected from the behavior observed for the isovector and isoscalar electric form factors, the proton electric form factor is consistently higher than the experimental results. The proton magnetic form factor agrees with the experiment for all $Q^{2}$ except the lowest two. This may be due to finite volume or residual excited state effects as discussed in Sec. V.

In Fig. 34 we show our results for the neutron form factors. The determination of $G_{E}^{n}\left(Q^{2}\right)$ directly from lattice QCD is very promising: We find good agreement with the experimental values but more importantly, at low $Q^{2}$, the errors from lattice QCD are smaller by up to a factor of 4 in some cases, allowing for a more precise description of its $Q^{2}$ dependence. The lattice QCD determination yields also accurate results for $G_{M}^{n}\left(Q^{2}\right)$ that are in agreement with experiment for $Q^{2}>0.2 \mathrm{GeV}^{2}$. At small $Q^{2}$ we observe the same discrepancy as that observed for the isovector case. Such an underestimation has also been seen for the induced pseudoscalar form factor where leading order chiral perturbation theory can show that it is due to multihadron state contributions with pions. Whether this is the explanation also for the neutron magnetic form factor remains an open question.

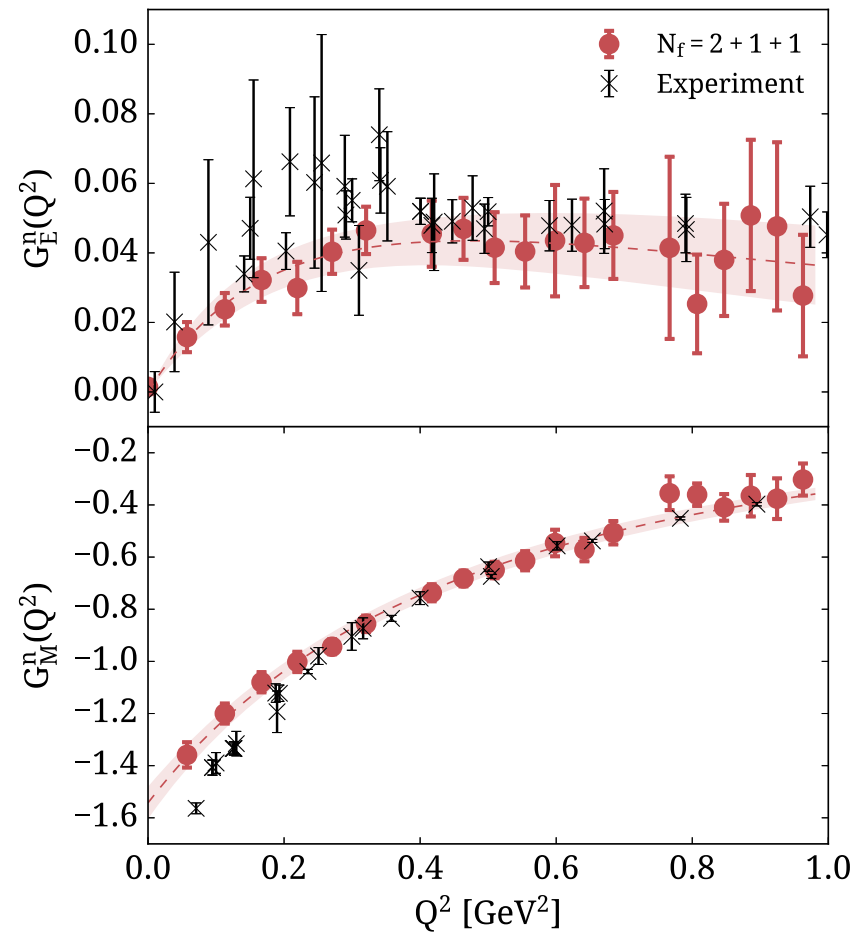

FIG. 34. Neutron electric (upper panel) and magnetic (lower panel) form factors as a function of $Q^{2}$. Filled circles show the lattice QCD results of this work and black crosses are experimental results taken from Refs. [4,46-59] for the case of the electric form factor and from Refs. [63-68] for the case of the magnetic form factor. The fits to our results use Eq. (21) for the electric form factor and Eq. (19) for the magnetic form factors.
Our results for the proton radii and magnetic moment, as extracted from the dipole fit, are

$$
\begin{aligned}
\sqrt{\left\langle r_{E}^{2}\right\rangle^{p}} & =0.742(13)(9)(14) \mathrm{fm}, \\
\mu_{p} & =2.43(9)(1)(3)\left(\begin{array}{l}
1 \\
0
\end{array}\right), \\
\sqrt{\left\langle r_{M}^{2}\right\rangle^{p}} & =0.710(26)(80)(6)\left(\begin{array}{l}
2 \\
0
\end{array}\right) \mathrm{fm} .
\end{aligned}
$$

The corresponding quantities for the neutron using the Galster-like parametrization for the electric and the dipole forms for the magnetic moment are

$$
\begin{aligned}
\left\langle r_{E}^{2}\right\rangle^{n} & =-0.074(16)(16)(8) \mathrm{fm}^{2}, \\
\mu_{n} & =-1.54(6)(2)(3), \\
\sqrt{\left\langle r_{M}^{2}\right\rangle^{n}} & =0.716(29)(44)(24) \mathrm{fm} .
\end{aligned}
$$

As already explained, the first error is statistical, the second is an estimate of the systematic due to the Ansätz chosen for the fit, and the third an estimate of excited state effects. We note here that disconnected contributions to $\left\langle r_{E}^{2}\right\rangle^{n}$ are nonnegligible. If we were to neglect them we would obtain $\left\langle r_{E}^{2}\right\rangle^{n \text {,conn }}=-0.063(15) \mathrm{fm}^{2}$, namely more than a $15 \%$ shift in the mean value, i.e., comparable to the other quoted systematic errors.

\section{SUMMARY AND CONCLUSIONS}

The nucleon electromagnetic Sachs form factors are computed using an $N_{f}=2+1+1$ ensemble of maximally twisted mass fermions with quark masses tuned to their physical values as well as an ensemble of $N_{f}=2$ twisted mass fermions simulated at a pion mass of $130 \mathrm{MeV}$. Comparing results calculated using $N_{f}=2$ and $N_{f}=2+$ $1+1$ twisted mass ensembles leads to the conclusion that no quenching effects are detected within the accuracy of the results that is within a couple of a percentage.

A main novelty of this work is the computation to an unprecedented accuracy of the disconnected light quark contributions, allowing us to extract the individual proton and neutron electromagnetic form factors. This is accomplished by using state-of-the-art techniques that combine hierarchical probing and deflation of the lowest eigenmodes and a large number of randomly distributed smeared point sources in order to suppress gauge noise. In particular, we find that disconnected contributions to the neutron electric form factor are non-negligible and need to be taken into account to bring agreement with the experimental values.

Excited states are thoroughly investigated using five sink-source time separations in the range of [0.96-1.60] fm allowing the identification of the ground state to good precision and the determination of a systematic error due to the excited states by comparing results from the plateau 
TABLE V. Our results for the electromagnetic radii and the magnetic moment using the $N_{f}=2+1+1$ ensemble for the isovector combination $(p-n)$, isoscalar $(p+n)$, the proton, and neutron. The first error is statistical, the second is a systematic due to the fit Ansätz, and the third a systematic due to excited states, derived as explained in the text.

\begin{tabular}{lcll}
\hline \hline & $\sqrt{\left\langle r_{E}^{2}\right\rangle}(\mathrm{fm})$ & \multicolumn{1}{c}{$\sqrt{\left\langle r_{M}^{2}\right\rangle}(\mathrm{fm})$} & $\mu$ \\
\hline$p-n$ & $0.796(19)(12)(12)$ & $0.712(27)(87)(5)$ & $3.97(15)(2)(5)$ \\
$p+n$ & $0.691(9)(7)(14)$ & $0.695(36)(80)(13)$ & $0.89(4)(3)(3)$ \\
$p$ & $0.742(13)(9)(14)$ & $0.710(26)(80)(6)$ & $2.43(9)(1)(3)$ \\
$n$ & $\left\langle r_{E}^{2}\right\rangle\left(\mathrm{fm}^{2}\right)$ & $0.716(29)(44)(24)$ & $-1.54(6)(2)(3)$ \\
& $-0.074(16)(16)(8)$ & & \\
\hline \hline
\end{tabular}

method with the two-state fit method. The summation method is used as a confirmation of the results extracted from the plateau and two-state fits.

Our values for the electric and magnetic rms radii as well as the magnetic moments for the isovector, isoscalar, proton, and neutron are collected in Table V. The results are extracted using the dipole Ansätz or the Galster-like parametrization and a systematic error on the parametrization used is extracted by comparing with the modelindependent z-expansion. Our result for the proton electric rms radius is underestimated due to the slower decay of $G_{E}^{p}\left(Q^{2}\right)$. Similarly there is an underestimation of the magnetic moments for the proton and neutron. The most plausible explanation for these remaining discrepancies may come from a combination of residual volume and multihadron contributions. Finite volume effects are investigated in this work by comparing two $N_{f}=2$ twisted mass ensembles with pion mass of $130 \mathrm{MeV}$ with the same lattice spacing but $L m_{\pi} \simeq 3$ and $L m_{\pi} \simeq 4$. Although we observe consistent results between these two volumes, we cannot exclude finite volume effects that may affect the magnetic form factor for small $Q^{2}$ values as well as the electric form factor. A slow convergence of the results as a function of the volume in combination with effects of multihadron states maybe difficult to detect. A theoretical investigation within chiral perturbation theory can shed light on multihadronic contributions. Furthermore, a study of a larger volume will also help to probe adequately volume effects. Thus, further studies are required to be able to take the infinite volume limit and make definite conclusions on the small $Q^{2}$ behavior of the magnetic form factor and on the slope of the electric form factor. Finite lattice spacing effects, although they are expected to be small, need to be investigated also. Before this program is completed one cannot make final statements on the two experimental results for the proton charge radius. The ETM Collaboration is generating further ensembles in order to enable the investigation of these issues that will require large computational resources.

\section{ACKNOWLEDGMENTS}

We would like to thank all members of ETMC for a very constructive and enjoyable collaboration. M. C. acknowledges financial support by the U.S. National Science Foundation under Grant No. PHY-1714407. This project has received funding from the Horizon 2020 research and innovation program of the European Commission under the Marie Skłodowska-Curie Grant Agreement No. 642069. $\mathrm{S}$. B. is supported by this program as well as from the project COMPLEMENTARY/0916/0015 funded by the Cyprus Research Promotion Foundation. The authors gratefully acknowledge the Gauss Centre for Supercomputing e.V. for funding the Project No. pr74yo by providing computing time on the GCS Supercomputer SuperMUC at Leibniz Supercomputing Centre. Results were obtained using Piz Daint at Centro Svizzero di Calcolo Scientifico (CSCS), via the project ID No. s702. We thank the staff of CSCS for access to the computational resources and for their constant support. This work also used computational resources from Extreme Science and Engineering Discovery Environment, which is supported by National Science Foundation Grant No. TG-PHY170022. This work used computational resources from the John von Neumann Institute for Computing on the Jureca system at the research center in Jülich, under Project ID No. ECY00.

\section{APPENDIX A: EXPRESSIONS RELATING NUCLEON VECTOR MATRIX ELEMENTS TO ELECTROMAGNETIC FORM FACTORS}

In this appendix we give a summary of the expressions relating the Sachs form factors $G_{E} \equiv G_{E}\left(Q^{2}\right)$ and $G_{M} \equiv$ $G_{M}\left(Q^{2}\right)$ to the ratio of three-point and two-point functions. The expressions are given for a general frame with initial (final) momentum $\vec{p}\left(\vec{p}^{\prime}\right)$ and initial (final) energy $E\left(E^{\prime}\right)$. All expressions are given in Euclidean space,

$$
\begin{aligned}
\Pi_{\mu}\left(\Gamma_{0}, \vec{p}^{\prime}, \vec{p}\right)= & \frac{-i C G_{E}}{2 m\left(4 m^{2}+Q^{2}\right)}\left(\left(p_{\mu}^{\prime}+p_{\mu}\right)\left[m\left(E^{\prime}+E+m\right)-p_{\rho}^{\prime} p_{\rho}\right]\right) \\
& +\frac{C G_{M}}{4 m^{2}\left(4 m^{2}+Q^{2}\right)}\left(\delta_{\mu 0}\left(4 m^{4}+m^{2} Q^{2}+4 m^{2} p_{\rho}^{\prime} p_{\rho}+Q^{2} p_{\rho}^{\prime} p_{\rho}\right)\right. \\
& +2 i m^{2} p_{\mu}^{\prime}\left(E^{\prime}-E\right)-2 i m^{3}\left(p_{\mu}^{\prime}+p_{\mu}\right)-i E Q^{2} p_{\mu}^{\prime}-i E^{\prime} Q^{2} p_{\mu} \\
& \left.-i m Q^{2}\left(p_{\mu}^{\prime}+p_{\mu}\right)-2 i m^{2} p_{\mu}\left(E^{\prime}-E\right)-2 i m p_{\rho}^{\prime} p_{\rho}\left(p_{\mu}^{\prime}+p_{\mu}\right)\right),
\end{aligned}
$$




$$
\begin{aligned}
\Pi_{\mu}\left(\Gamma_{k}, \vec{p}^{\prime}, \vec{p}\right)= & \frac{-C G_{E}}{2 m\left(4 m^{2}+Q^{2}\right)}\left(m^{2} \varepsilon_{\mu k 0 \rho}\left(p_{\rho}^{\prime}-p_{\rho}\right)-i \varepsilon_{\mu k \rho \sigma} p_{\rho}^{\prime} p_{\sigma}\left(E^{\prime}+E\right)\right. \\
& \left.+\varepsilon_{\mu 0 \rho \sigma} p_{\rho}^{\prime} p_{\sigma}\left(p_{k}^{\prime}+p_{k}\right)-\varepsilon_{\mu k 0 \rho} p_{\sigma}^{\prime} p_{\sigma}\left(p_{\rho}^{\prime}-p_{\rho}\right)\right)-\frac{C G_{M}}{4 m^{2}\left(4 m^{2}+Q^{2}\right)}\left(m \varepsilon_{\mu k 0 \rho}\left(p_{\rho}^{\prime}-p_{\rho}\right)\left(2 m^{2}+Q^{2}\right)\right. \\
& \left.+2 i m \varepsilon_{\mu k \rho \sigma} p_{\rho}^{\prime} p_{\sigma}\left(2 m+E^{\prime}+E+\frac{Q^{2}}{2 m}\right)-2 m \varepsilon_{\mu 0 \rho \sigma} p_{\rho}^{\prime} p_{\sigma}\left(p_{k}^{\prime}+p_{k}\right)+2 m \varepsilon_{\mu k 0 \rho} p_{\sigma}^{\prime} p_{\sigma}\left(p_{\rho}^{\prime}-p_{\rho}\right)\right)
\end{aligned}
$$

where $C$ is a kinematic factor given by

$$
C=\frac{2 m}{E\left(E\left(\vec{p}^{\prime}\right)+m\right)} \sqrt{\frac{E\left(E\left(\vec{p}^{\prime}\right)+m\right)}{E\left(\vec{p}^{\prime}\right)(E+m)}}
$$

$$
\begin{gathered}
\Pi_{i}\left(\Gamma_{0}, \vec{p}\right)=C \frac{p_{i}}{2 m} G_{E}\left(Q^{2}\right), \\
\Pi_{i}\left(\Gamma_{k}, \vec{p}\right)=C \frac{\epsilon_{i j k} p_{j}}{2 m} G_{M}\left(Q^{2}\right),
\end{gathered}
$$

In the case where $\vec{p}^{\prime}=\overrightarrow{0}$ the expressions simplify as and follows:

$$
\Pi_{0}\left(\Gamma_{0}, \vec{p}\right)=C \frac{E+m}{2 m} G_{E}\left(Q^{2}\right)
$$

$$
C=\sqrt{\frac{2 m^{2}}{E(E+m)}}
$$

\section{APPENDIX B: NUMERICAL RESULTS FOR THE ELECTROMAGNETIC FORM FACTORS}

TABLE VI. Results for the electromagnetic form factors using the cB211.072.64 ensemble for the isovector combination $G_{E, M}^{p}-G_{E, M}^{n}$, for the proton $G_{E, M}^{p}$, and neutron $G_{E, M}^{n}$, including the disconnected contributions for the latter two form factors.

\begin{tabular}{lllllll}
\hline \hline$Q^{2}\left(\mathrm{GeV}^{2}\right)$ & $G_{E}^{p-n}\left(Q^{2}\right)$ & $G_{E}^{p}\left(Q^{2}\right)$ & $G_{E}^{n}\left(Q^{2}\right)$ & $G_{M}^{u-d}\left(Q^{2}\right)$ & $G_{M}^{p}\left(Q^{2}\right)$ & $G_{M}^{n}\left(Q^{2}\right)$ \\
\hline 0.000 & $0.997(3)$ & $0.998(2)$ & $0.001(1)$ & $\mathrm{NA}$ & $\mathrm{NA}$ & NA \\
0.057 & $0.858(10)$ & $0.874(6)$ & $0.016(5)$ & $3.516(101)$ & $2.156(62)$ & $-1.361(43)$ \\
0.113 & $0.752(11)$ & $0.775(8)$ & $0.023(5)$ & $3.105(78)$ & $1.903(48)$ & $-1.202(33)$ \\
0.167 & $0.662(14)$ & $0.694(9)$ & $0.032(7)$ & $2.801(80)$ & $1.719(47)$ & $-1.082(35)$ \\
0.219 & $0.601(17)$ & $0.631(10)$ & $0.030(8)$ & $2.583(82)$ & $1.580(50)$ & $-1.003(35)$ \\
0.270 & $0.534(14)$ & $0.575(9)$ & $0.040(7)$ & $2.430(62)$ & $1.485(38)$ & $-0.945(26)$ \\
0.320 & $0.482(16)$ & $0.529(11)$ & $0.046(7)$ & $2.224(70)$ & $1.367(43)$ & $-0.857(29)$ \\
0.417 & $0.405(23)$ & $0.450(16)$ & $0.045(10)$ & $1.943(78)$ & $1.200(48)$ & $-0.743(32)$ \\
0.464 & $0.374(21)$ & $0.420(15)$ & $0.047(9)$ & $1.789(71)$ & $1.104(44)$ & $-0.684(29)$ \\
0.510 & $0.363(25)$ & $0.404(18)$ & $0.041(11)$ & $1.655(69)$ & $1.012(42)$ & $-0.644(31)$ \\
0.554 & $0.345(27)$ & $0.385(21)$ & $0.040(11)$ & $1.610(84)$ & $0.994(50)$ & $-0.616(37)$ \\
0.598 & $0.310(43)$ & $0.351(33)$ & $0.041(16)$ & $1.472(127)$ & $0.923(79)$ & $-0.549(51)$ \\
0.642 & $0.291(33)$ & $0.336(27)$ & $0.045(13)$ & $1.495(109)$ & $0.926(67)$ & $-0.570(44)$ \\
0.684 & $0.263(34)$ & $0.308(27)$ & $0.046(13)$ & $1.386(112)$ & $0.866(71)$ & $-0.521(44)$ \\
0.767 & $0.239(60)$ & $0.283(48)$ & $0.043(27)$ & $0.893(149)$ & $0.532(90)$ & $-0.361(65)$ \\
0.807 & $0.250(39)$ & $0.278(31)$ & $0.028(15)$ & $0.942(101)$ & $0.583(62)$ & $-0.361(43)$ \\
0.847 & $0.213(43)$ & $0.249(35)$ & $0.035(16)$ & $1.006(117)$ & $0.605(70)$ & $-0.402(51)$ \\
0.886 & $0.158(61)$ & $0.203(51)$ & $0.048(22)$ & $1.028(211)$ & $0.662(135)$ & $-0.367(80)$ \\
0.925 & $0.150(58)$ & $0.205(50)$ & $0.056(24)$ & $0.891(190)$ & $0.539(115)$ & $-0.353(79)$ \\
0.963 & $0.172(48)$ & $0.200(41)$ & $0.029(18)$ & $0.765(153)$ & $0.461(94)$ & $-0.302(61)$ \\
\hline \hline
\end{tabular}


[1] J. C. Bernauer et al. (A1 Collaboration), Phys. Rev. C 90, 015206 (2014).

[2] V. Punjabi, C. F. Perdrisat, M. K. Jones, E. J. Brash, and C. E. Carlson, Eur. Phys. J. A 51, 79 (2015).

[3] R. Pohl et al., Nature (London) 466, 213 (2010).

[4] J. Golak, G. Ziemer, H. Kamada, H. Witala, and W. Gloeckle, Phys. Rev. C 63, 034006 (2001).

[5] C. Alexandrou et al., Phys. Rev. D 98, 054518 (2018).

[6] C. Alexandrou, M. Constantinou, K. Hadjiyiannakou, K. Jansen, C. Kallidonis, G. Koutsou, and A. Vaquero AvilésCasco, Phys. Rev. D 97, 094504 (2018).

[7] C. Alexandrou, M. Constantinou, K. Hadjiyiannakou, K. Jansen, C. Kallidonis, G. Koutsou, and A. Vaquero AvilesCasco, Phys. Rev. D 96, 034503 (2017).

[8] S. Galster, H. Klein, J. Moritz, K. H. Schmidt, D. Wegener, and J. Bleckwenn, Nucl. Phys. B32, 221 (1971).

[9] R. J. Hill and G. Paz, Phys. Rev. D 82, 113005 (2010).

[10] A. Stathopoulos, J. Laeuchli, and K. Orginos, arXiv: 1302.4018.

[11] A. S. Gambhir, A. Stathopoulos, and K. Orginos, SIAM J. Sci. Comput. 39, A532 (2017).

[12] S. Capitani, M. Della Morte, D. Djukanovic, G. von Hippel, J. Hua, B. Jager, B. Knippschild, H. B. Meyer, T. D. Rae, and H. Wittig, Phys. Rev. D 92, 054511 (2015).

[13] J. R. Green, J. W. Negele, A. V. Pochinsky, S. N. Syritsyn, M. Engelhardt, and S. Krieg, Phys. Rev. D 90, 074507 (2014).

[14] N. Hasan, J. Green, S. Meinel, M. Engelhardt, S. Krieg, J. Negele, A. Pochinsky, and S. Syritsyn, Phys. Rev. D 97, 034504 (2018).

[15] K.-I. Ishikawa, Y. Kuramashi, S. Sasaki, N. Tsukamoto, A. Ukawa, and T. Yamazaki (PACS Collaboration), Phys. Rev. D 98, 074510 (2018).

[16] C. Alexandrou, S. Gusken, F. Jegerlehner, K. Schilling, and R. Sommer, Nucl. Phys. B414, 815 (1994).

[17] S. Gusken, Nucl. Phys. B, Proc. Suppl. 17, 361 (1990).

[18] C. Alexandrou et al. (European Twisted Mass Collaboration), Phys. Rev. D 78, 014509 (2008).

[19] M. Albanese et al. (APE Collaboration), Phys. Lett. B 192, 163 (1987).

[20] C. Alexandrou, M. Constantinou, S. Dinter, V. Drach, K. Jansen, C. Kallidonis, and G. Koutsou, Phys. Rev. D 88, 014509 (2013).

[21] C. Alexandrou, M. Brinet, J. Carbonell, M. Constantinou, P. A. Harraud, P. Guichon, K. Jansen, T. Korzec, and M. Papinutto, Phys. Rev. D 83, 094502 (2011).

[22] C. Alexandrou, G. Koutsou, J. W. Negele, and A. Tsapalis, Phys. Rev. D 74, 034508 (2006).

[23] S. Bacchio, C. Alexandrou, and J. Finkerath, EPJ Web Conf. 175, 02002 (2018).

[24] S. Bacchio, C. Alexandrou, J. Finkenrath, A. Frommer, K. Kahl, and M. Rottmann, Proc. Sci., LATTICE2016 (2016) 259 [arXiv:1611.01034].

[25] C. Alexandrou, S. Bacchio, J. Finkenrath, A. Frommer, K. Kahl, and M. Rottmann, Phys. Rev. D 94, 114509 (2016).

[26] W. Wilcox, Noise methods for flavor singlet quantities, in Numerical Challenges in Lattice Quantum Chromodynamics, edited by A. Frommer, T. Lippert, B. Medeke, and K. Schilling (Springer, Berlin, Heidelberg, 1999), pp. 127-141, https://doi.org/10.1007/978-3-642-58333-9_10.
[27] J. Green, S. Meinel, M. Engelhardt, S. Krieg, J. Laeuchli, J. Negele, K. Orginos, A. Pochinsky, and S. Syritsyn, Phys. Rev. D 92, 031501 (2015).

[28] J. Green, N. Hasan, S. Meinel, M. Engelhardt, S. Krieg, J. Laeuchli, J. Negele, K. Orginos, A. Pochinsky, and S. Syritsyn, Phys. Rev. D 95, 114502 (2017).

[29] C. McNeile and C. Michael (UKQCD Collaboration), Phys. Rev. D 73, 074506 (2006).

[30] C. Alexandrou, M. Constantinou, V. Drach, K. Hadjiyiannakou, K. Jansen, G. Koutsou, A. Strelchenko, and A. Vaquero, Comput. Phys. Commun. 185, 1370 (2014).

[31] C. Alexandrou, M. Constantinou, K. Hadjiyiannakou, K. Jansen, C. Kallidonis, G. Koutsou, and A. Vaquero AvilesCasco, Phys. Rev. D 96, 054507 (2017).

[32] C. Alexandrou et al., Phys. Rev. D 95, 114514 (2017); 96, 099906(E) (2017).

[33] C. Alexandrou, M. Constantinou, K. Hadjiyiannakou, K. Jansen, C. Kallidonis, G. Koutsou, A. Vaquero AvilésCasco, and C. Wiese, Phys. Rev. Lett. 119, 142002 (2017).

[34] R. Frezzotti and G. C. Rossi, J. High Energy Phys. 08 (2004) 007.

[35] R. Frezzotti and G. C. Rossi, Nucl. Phys. B, Proc. Suppl. 128, 193 (2004).

[36] A. Abdel-Rehim et al. (ETM Collaboration), Phys. Rev. D 95, 094515 (2017).

[37] L. Maiani, G. Martinelli, M. L. Paciello, and B. Taglienti, Nucl. Phys. B293, 420 (1987).

[38] S. Capitani, M. Della Morte, G. von Hippel, B. Jager, A. Juttner, B. Knippschild, H. B. Meyer, and H. Wittig, Phys. Rev. D 86, 074502 (2012).

[39] M. J. Savage, P. E. Shanahan, B. C. Tiburzi, M. L. Wagman, F. Winter, S. R. Beane, E. Chang, Z. Davoudi, W. Detmold, and K. Orginos, Phys. Rev. Lett. 119, 062002 (2017).

[40] C. Alexandrou, M. Constantinou, and H. Panagopoulos (ETM Collaboration), Phys. Rev. D 95, 034505 (2017).

[41] C. Alexandrou, M. Constantinou, T. Korzec, H. Panagopoulos, and F. Stylianou, Phys. Rev. D 83, 014503 (2011).

[42] R. S. Sufian, Y.-B. Yang, A. Alexandru, T. Draper, J. Liang, and K.-F. Liu, Phys. Rev. Lett. 118, 042001 (2017).

[43] O. Bar, Phys. Rev. D 99, 054506 (2019).

[44] C. F. Perdrisat, V. Punjabi, and M. Vanderhaeghen, Prog. Part. Nucl. Phys. 59, 694 (2007).

[45] W. M. Alberico, S. M. Bilenky, C. Giunti, and K. M. Graczyk, Phys. Rev. C 79, 065204 (2009).

[46] J. Becker et al., Eur. Phys. J. A 6, 329 (1999).

[47] T. Eden et al., Phys. Rev. C 50, R1749 (1994).

[48] M. Meyerhoff et al., Phys. Lett. B 327, 201 (1994).

[49] I. Passchier et al., Phys. Rev. Lett. 82, 4988 (1999).

[50] G. Warren et al. (Jefferson Lab E93-026 Collaboration), Phys. Rev. Lett. 92, 042301 (2004).

[51] H. Zhu et al. (E93026 Collaboration), Phys. Rev. Lett. 87, 081801 (2001).

[52] B. Plaster et al. (Jefferson Laboratory E93-038 Collaboration), Phys. Rev. C 73, 025205 (2006).

[53] R. Madey et al. (E93-038 Collaboration), Phys. Rev. Lett. 91, 122002 (2003). 
[54] D. Rohe et al., Phys. Rev. Lett. 83, 4257 (1999).

[55] J. Bermuth et al., Phys. Lett. B 564, 199 (2003).

[56] D. I. Glazier et al., Eur. Phys. J. A 24, 101 (2005).

[57] C. Herberg et al., Eur. Phys. J. A 5, 131 (1999).

[58] R. Schiavilla and I. Sick, Phys. Rev. C 64, 041002 (2001).

[59] M. Ostrick et al., Phys. Rev. Lett. 83, 276 (1999).

[60] T. Sato, D. Uno, and T. S. H. Lee, Phys. Rev. C 67, 065201 (2003).

[61] E. Shintani, K.-I. Ishikawa, Y. Kuramashi, S. Sasaki, and T. Yamazaki, Phys. Rev. D 99, 014510 (2019).

[62] R. S. Sufian, Y.-B. Yang, J. Liang, T. Draper, and K.-F. Liu, Phys. Rev. D 96, 114504 (2017).
[63] B. Anderson et al. (Jefferson Lab E95-001 Collaboration), Phys. Rev. C 75, 034003 (2007).

[64] H. Gao et al., Phys. Rev. C 50, R546 (1994).

[65] H. Anklin et al., Phys. Lett. B 336, 313 (1994).

[66] H. Anklin et al., Phys. Lett. B 428, 248 (1998).

[67] G. Kubon et al., Phys. Lett. B 524, 26 (2002).

[68] R. Alarcon (BLAST Collaboration), Eur. Phys. J. A 32, 477 (2007).

[69] P. J. Mohr, D. B. Newell, and B. N. Taylor, Rev. Mod. Phys. 88, 035009 (2016).

[70] C. Patrignani et al. (Particle Data Group), Chin. Phys. C 40, 100001 (2016). 